\title{
HOLES AND SHELLS IN THE INTERSTELLAR MEDIUM OF THE NEARBY DWARF GALAXY IC 2574
}

\author{
FABIAN WALTER ${ }^{1}$ \\ Radioastronomisches Institut der Universität, Auf dem Hügel 71, D-53121 Bonn, Germany \\ AND \\ ELIAS BRINKS \\ Departamento de Astronomía, Apdo. Postal 144, Guanajuato, GTO 36000, Mexico; National Radio Astronomy Observatory, P.O. Box O, \\ Socorro, NM 87801; ebrinks@ astro.ugto.mx \\ Received 1998 March 10; accepted 1999 March 30
}

\begin{abstract}
We present $\mathrm{H}$ I synthesis observations of the nearby dwarf galaxy IC 2574 (a member of the M81 group of galaxies) made with the $\mathrm{NRAO}^{2}$ Very Large Array (VLA) in its $\mathrm{B}, \mathrm{C}$, and D configurations at high spatial and velocity resolution $\left(95 \mathrm{pc} \times 2.6 \mathrm{~km} \mathrm{~s}^{-1}\right)$. In addition, we present optical broad- and narrowband images obtained with the $2.2 \mathrm{~m}$ telescope of the Calar Alto Observatory. The VLA H I observations show a stunning amount of detail in the form of 48 mostly expanding $\mathrm{H}$ I shells and holes in its neutral interstellar medium. These features range in size from about $100 \mathrm{pc}$ (a limit set by the size of the beam) to about $1000 \mathrm{pc}$, dominating the appearance of the $\mathrm{H}$ I surface brightness map. Their dynamics clearly influence the velocity field of IC 2574. In addition to well defined holes, some large scale $(>1000 \mathrm{pc})$ coherent features are visible in the channel maps. They are probably the remainder of an older shell population. Current star formation, as traced by $\mathrm{H} \alpha$ emission, is predominantly found along the rims of the larger $\mathrm{H}$ I holes, suggesting propagating star formation. On linear scales of $\approx 95$ pc, star formation occurs if the $\mathrm{H}$ I surface density reaches values higher than $10^{21} \mathrm{~cm}^{-2}$. The radial expansion of the $\mathrm{H}$ I holes $\left(8-12 \mathrm{~km} \mathrm{~s}^{-1}\right)$, the indicative ages (10-60 Myr) and the energy requirements for their formation $\left(10^{50}-10^{53}\right.$ ergs) can be understood in terms of the combined effects of stellar winds and multiple supernova explosions of the most massive stars formed during a recent phase of active star formation.

The scale height of the $\mathrm{H}$ I layer is found to be $\approx 350 \mathrm{pc}$, considerably thicker than that in massive disk galaxies. This is due to a lower gravitational potential (for the same observed one-dimensional velocity dispersion of $\left.\approx 7 \mathrm{~km} \mathrm{~s}^{-1}\right)$. This puffed-up disk implies a lower $\mathrm{H}$ I volume density $\left(0.15 \mathrm{~cm}^{-3}\right)$. This fact, combined with the reduced gravitational pull and solid-body rotation throughout the disk, explains why the diameter distribution in dwarf galaxies extends to substantially larger values than in spiral galaxies. A comparison with other galaxies shows that the energies needed to create these structures are the same for all types of galaxies, at least to first order. The overall statistical properties of the $\mathrm{H}$ I holes and shells in galaxies show clear trends with Hubble type (or rather mass), such as in their diameter distribution, expansion velocities, and ages.
\end{abstract}

Key words: galaxies: individual (IC 2574) — galaxies: ISM — ISM: structure — radio emission lines

\section{INTRODUCTION}

Some twenty years ago, Heiles $(1979,1984,1990)$ discovered large, shell-like structures in the neutral hydrogen (H I) distribution of our Galaxy by inspecting maps made in the $21 \mathrm{~cm}$ line. Since his discovery, a wealth of observations obtained with powerful synthesis radio telescopes such as the Very Large Array (VLA) or the Westerbork Synthesis Radio Telescope (WSRT) revealed similar structures in our nearest neighbors. Prominent examples are M31 (Brinks 1981; Brinks \& Bajaja 1986), M33 (Deul \& den Hartog 1990), Holmberg II (Puche et al. 1992, hereafter PWBR92), the galaxies M101 and NGC 6946 (Kamphuis 1993), and IC 10 (Wilcots \& Miller 1998). The latest, impressive additions to this list are the SMC (Staveley-Smith et al. 1997) and

\footnotetext{
${ }^{1}$ Visiting Astronomer, German-Spanish Astronomical Centre, Calar Alto, operated by the Max-Planck-Institut für Astronomie (Heidelberg) jointly with the Spanish Comisión Nacional de Astronomía.

${ }^{2}$ The National Radio Astronomy Observatory is a facility of the National Science Foundation operated under cooperative agreement by Associated Universities, Inc.
}

LMC (Kim et al. 1998). All these observations indicate that the interstellar medium (ISM) of medium- to late-type galaxies is dominated by features that are variously described as shells, rings, holes, loops, bubbles, or cavities.

Obviously, one of the basic questions is which physical process is able to produce these observed structures. The general picture that has emerged is that they are the result of combined stellar winds and supernova explosions produced by young stellar associations. For review articles on that topic, see Tenorio-Tagle \& Bodenheimer (1988) and van der Hulst (1996 and references therein). Based on a simple model of holes created by O and B stars, Oey \& Clarke (1997) successfully predict the observed number distribution of SMC holes, lending support to this hypothesis. It should be noted, however, that this general picture is not without its flaws. Searches for the remnant stellar populations of the $\mathrm{OB}$ associations thought to be responsible for some of the holes in Ho II have not led to the expected result (Rhode, Salzer, \& Westpfhal 1999). And in the case of the largest observed shells, these seem to have energy requirements surpassing the output of stellar winds and supernovae. To explain those structures an alternative mechanism was proposed, namely, the infall of gas clouds 
TABLE 1

GENERAL INFORMATION ON IC $2574^{\mathrm{a}}$

\begin{tabular}{|c|c|}
\hline Parameter & Value \\
\hline Right ascension $(1950.0)^{\mathbf{b}}$ & 102441.3 \\
\hline Declination $(1950.0)^{b}$ & 684018 \\
\hline Adopted distance $(\mathrm{Mpc})^{\mathrm{c}}$ & 3.2 \\
\hline Inclination $(\mathrm{deg})^{\mathrm{d}} \ldots \ldots$. & $77 \pm 3$ \\
\hline Scale (arcsec to pc) & $15 . \overline{5}$ \\
\hline Apparent magnitude $(B)(\mathrm{mag})^{\mathrm{e}}$. & 10.8 \\
\hline 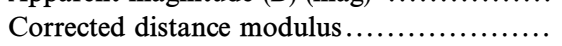 & $m_{B}-M_{B}=27.6$ \\
\hline Corrected absolute magnitude $(B)(\mathrm{mag}) \ldots \ldots$. & $-16.8^{D}$ \\
\hline Blue luminosity $L_{B}\left(L_{B \odot}\right) \ldots \ldots \ldots \ldots \ldots \ldots \ldots \ldots \ldots \ldots \ldots$ & $7.8 \times 10^{8}$ \\
\hline H I mass $M_{\mathrm{HI}}\left(M_{\odot}\right)^{\mathrm{f}} \ldots \ldots \ldots \ldots \ldots \ldots \ldots \ldots \ldots \ldots \ldots \ldots \ldots \ldots \ldots$ & $9.0 \times 10^{8}$ \\
\hline H I mass to blue light ratio $\left(M_{\odot} / L_{B \odot}\right) \ldots \ldots \ldots$ & 1.15 \\
\hline
\end{tabular}

NoTE.-Units of right ascension are hours, minutes, and seconds, and units of declination are degrees, arcminutes, and arcseconds.

${ }^{a}$ Other names: UGC 5666, DDO 81, VII Zw 330.

b Dressel \& Condon 1976.

${ }^{\mathrm{c}}$ See discussion in Puche et al. 1992.

d Martimbeau et al. 1994.

e de Vaucouleurs et al. 1993 (RC3).

f This study.

(see Tenorio-Tagle et al. 1987 for a numerical simulation and van der Hulst \& Sancisi 1988 for observational evidence in the case of one of the largest holes in M101). And recently, even Gamma Ray Bursters (GRBs) have been suggested as a possible explanation for the largest $\mathrm{H}$ i holes (Efremov, Elmegreen, \& Hodge 1998; Loeb \& Perna 1998).

The idea that supernovae deposit large amounts of energy into the ISM is not new. Cox \& Smith (1974) already discussed the idea that the energy released by supernovae sets up tunnels of hot gas in the ISM. This picture was a substantial modification of the Field, Goldsmith, \& Habing (1969) model which consists of a two-phase medium. The ideas proposed by Cox \& Smith were incorporated within the model elaborated by McKee \& Ostriker (1977), who suggested that the ISM has three distinct phases: cool $\left(T \approx 10^{2} \mathrm{~K}\right)$, warm $\left(T \approx 10^{4} \mathrm{~K}\right)$, and hot $\left(T \approx 10^{6} \mathrm{~K}\right)$, all in pressure equilibrium. Various refinements have been proposed, but this picture basically still stands.

Knowing how much kinetic energy is transferred in expanding shells and filaments is of direct importance for assessing the energy balance in the ISM. In addition to an analytical approach, various authors are putting forward numerical simulations (e.g., Palouš, Franco, \& TenorioTagle 1990; Silich et al. 1996; Palouš, Tenorio-Tagle, \& Franco 1994), in order to model both the evolution of the shape of the $\mathrm{H}$ I shells in various environments and the fragmentation of matter on the rim of the shells, and to investigate induced star formation. This is important not only for tracking down the mechanisms that produce the $\mathrm{H}$ I shells and filaments, but also for determining what fraction of the ISM breaks out into the halo and might eventually rain back onto the disk.

Our aim is to investigate the properties of the $\mathrm{H}$ I shells and bubbles in the ISM of galaxies. Intimately linked to this issue are the questions of when fragmentation on the rim of the shells starts and what drives star formation. Unfortunately, the determination of the characteristics of $\mathrm{H} \mathrm{I}$ shells in our Galaxy is not straightforward because of our unfavorable location within the disk. Looking at the nearest spiral galaxies (e.g., M31, M33, M101, NGC 6946) does not improve matters all that much since the structures in their ISM are strongly modified by spiral density waves and differential rotation, both effects dramatically influencing the shape and formation of holes and shells. Therefore, we decided to direct our attention to nearby dwarf galaxies instead. Dwarfs are slow rotators, generally show solidbody rotation, and lack density waves. This implies that once features such as shells have formed, they will not be deformed by galactic shear and will therefore tend to be long lived. Moreover, the overall gravitational potential of a dwarf is much smaller than that of a normal spiral. The same amount of energy input into a star-forming region thus has a more pronounced impact on the overall appearance of the ISM, as shown by PWBR92. Since dwarf galaxies also tend to have a much thicker disk compared to massive spirals (e.g., Staveley-Smith, Davies, \& Kinman 1992), the H I volume density of dwarf galaxies is lower, again facilitating the creation of large holes. And because of their smaller size, the probability that they will suffer impacts from infalling clouds is lower compared with that for larger galaxies. Despite all these arguments in favor, only very few such $\mathrm{H}$ I studies of dwarf galaxies have been published. Besides our two neighbors, the SMC and LMC, a detailed analysis was performed in only one case, that of the dwarf galaxy Holmberg II (PWBR92), member of the M81 group.

In this paper, we concentrate on IC 2574, a gas-rich dwarf galaxy in the same group. Table 1 summarizes some general information on this object. IC 2574 (=UGC $5666=$ DDO 81 , also sometimes referred to as Coddington's Nebula) has been studied by several authors in the past. Observations with single-dish telescopes in the $21 \mathrm{~cm}$ line of neutral hydrogen (H I) were published by Rots (1980) and Huchtmeier \& Richter (1988), and the first study using an interferometer was made by Seielstad \& Wright (1973). A recent H I study, concentrating on the dynamics and contribution to the total mass by dark matter was performed by Martimbeau, Carignan, \& Roy (1994, hereafter MCR94), who observed the galaxy with the WSRT. Recent broadband optical studies were published by Tikhonov et al. (1991). Studies of $\mathrm{H}$ II regions in IC 2574 have been presented by Miller \& Hodge (1994; hereafter MH94); Kennicutt (1988); and Arsenault \& Roy (1986). Spectroscopy of some prominent H II regions was performed by Miller \& Hodge (1996) and $\mathrm{H} \alpha$ velocity fields were recently obtained by Tomita et al. (1998). In addition, four Wolf-Rayet stars were discovered by Drissen, Roy, \& Moffat (1993).

We present $\mathrm{H}$ I observations made with the NRAO VLA in its B-, C-, \& D-array configurations, supplemented by optical observations obtained at the Calar Alto Observatory. The VLA data were originally obtained by Puche, Brinks, and Westpfahl with the intention of following up on their work on Ho II. So far, only a low-resolution integrated H I map was published by Puche \& Westpfahl (1994). This paper presents the VLA data in complete form and compares them with optical observations. We recalibrated the original data to produce far superior maps using the latest calibration and imaging techniques within AIPS. The data reduction and results are discussed in $\S \S 2$ and 3. In $\S 4$, a catalog of $\mathrm{H}$ I holes and shells is presented. Section 5 discusses the relation between $\mathrm{H}$ I and $\mathrm{H} \alpha$ features. A general discussion is given in $\S 6$, and a summary of the results is presented in $\S 7$. 


\section{OBSERVATIONS}

\subsection{Radio Observations}

IC 2574 was observed with the VLA in B, C, and D configurations. Part of the D-array observations were affected by solar interference, which was removed by deleting $(u, v)$ spacings shorter than typically $0.4 \mathrm{k} \lambda$ and editing out bad time ranges. In total, $17 \mathrm{hr}$ were spent on source, divided into $4 \mathrm{hr}$ for $\mathrm{D}, 2.5 \mathrm{hr}$ in $\mathrm{C}$, and $10.5 \mathrm{hr}$ in $\mathrm{B}$ configuration (see Table 2 for a detailed description of the VLA observations). The longer observing time in B array only partly compensates for the lower surface brightness sensitivity in this extended configuration. The absolute flux calibration was determined by observing $1328+307$ (3C 286) for approximately 20 minutes during each observing run, assuming a flux density of $14.73 \mathrm{Jy}$, according to the scale of Baars et al. (1977). This calibrator was also used to derive the complex bandpass corrections. The nearby calibrators $1031+567$ and $0945+664$ were used as secondary amplitude and phase calibrators and their fluxes were determined to be $1.80 \mathrm{Jy}$ and $2.22 \mathrm{Jy}$, respectively. Since the systemic velocity of IC $2574\left(\approx 53 \mathrm{~km} \mathrm{~s}^{-1}\right)$ is close in velocity to $\mathrm{H} \mathrm{I}$ emission from our Galaxy, each calibrator observation was split in two parts to form a pair of observations, one part having the velocity shifted by $+300 \mathrm{~km} \mathrm{~s}^{-1}$ and the other by $-300 \mathrm{~km} \mathrm{~s}^{-1}$ to avoid contamination by Galactic emission. In the course of the calibration these observations were then averaged to give interpolated amplitude and phase corrections. IC 2574 was observed using a $1.56 \mathrm{MHz}$ bandwidth centered at a heliocentric velocity of $38 \mathrm{~km} \mathrm{~s}^{-1}$. This band was divided into 128 channels, resulting in a velocity resolution of $2.58 \mathrm{~km} \mathrm{~s}^{-1}$ after on-line Hanning smoothing.

The data for each array were edited and calibrated separately with the AIPS package. ${ }^{3}$ The $(u, v)$ data were inspected and bad data points caused by either interference or cross talk between antennae removed, after which the data were calibrated. We Fourier-transformed our B-, C-,

${ }^{3}$ The Astronomical Image Processing System (AIPS) was developed by the NRAO. and D-configuration observations separately to assess their quality; subsequently we combined all data to form a single data set which was used for mapping. All the results presented in this paper are based on this combined data set.

Channels with velocities lower than $-40 \mathrm{~km} \mathrm{~s}^{-1}$ and higher than $+136 \mathrm{~km} \mathrm{~s}^{-1}$ were found to be line free; these channels determined the continuum emission that was subtracted in the $(u, v)$ plane. The same channels were used to produce a radio continuum map of IC 2574.

After that, two sets of data cubes $(1024 \times 1024$ pixels $\times 68$ channels each) were produced using the task IMAGR in AIPS, each of them CLEANed to a level of 2 times the rms noise (Högbom 1974; Clark 1980). The first data set was made with natural weighting, leading to a resolution of 12 ". $4 \times 11.9$ and emphasizing large-scale structures. A second cube was produced using the ROBUST weighting scheme (Briggs 1995). This scheme achieves the high sensitivity of natural weighting combined with a well-behaved synthesized beam at a resolution close to that of uniform weighting. To obtain the optimum ROBUST value for our observation, the ROBUST parameter space was searched by producing maps in the sensitive regime of $-2<$ ROBUST $<2$ and measuring the rms noise and beam size for each map. Eventually, we chose a value of ROBUST $=0.25$, resulting in a beam size of 6.4 $\times 5.9$ and an rms noise of $0.7 \mathrm{mJy}^{\prime \prime}$ beam $^{-1}$. A comparison with the beam size of the high-resolution uniform map (4".7 $\times 4$ ".3) and the rms noise of the low-noise, natural map $(\approx 10 \%$ increase in noise) shows the strength of this mapping algorithm. Unless otherwise mentioned, the results presented in the following are based on this ROBUST cube.

Although IC 2574 is situated at a Galactic latitude of 43.6 , some of the channels were clearly confused by Galactic emission (in particular between +10 and -10 ). Since Galactic emission shows its presence on predominantly large scales, we were able to remove the most prominent emission features by blanking the $(u, v)$ data out to $0.1 \mathrm{k} \lambda$ in these channels.

To separate real emission from the noise, the following procedure was applied: the natural-weighted data cube was convolved to a circular beam with a FWHM of $45^{\prime \prime}$. The

TABLE 2

SETUP OF THE VLA DURING THE OBSERVATIONS

\begin{tabular}{|c|c|c|c|}
\hline Parameter & B & $\mathrm{C}$ & $\mathrm{D}$ \\
\hline Baselines .................. & $\begin{array}{l}1-54 \mathrm{k} \lambda \\
(0.21-11.4 \mathrm{~km})\end{array}$ & $\begin{array}{l}0.34-16 \mathrm{k} \lambda \\
(0.073-3.4 \mathrm{~km})\end{array}$ & $\begin{array}{l}0.166-4.9 \mathrm{k} \lambda \\
(0.035-1.03 \mathrm{~km})\end{array}$ \\
\hline Date of observation ........ & $\begin{array}{l}1992 \text { Jan } 18 \\
1992 \text { Jan } 19\end{array}$ & 1992 Apr 20 & $\begin{array}{l}1991 \text { Mar } 3 \\
1992 \text { Sep } 3\end{array}$ \\
\hline Total time on source...... & 647 minutes & 133 minutes & 245 minutes \\
\hline Total bandwidth .......... & & $1.56 \mathrm{Mhz}$ & \\
\hline Number of channels ...... & & 128 & \\
\hline Velocity resolution ........ & & $2.58\left(\mathrm{~km} \mathrm{~s}^{-1}\right)$ & \\
\hline Central velocity............. & & $38\left(\mathrm{~km} \mathrm{~s}^{-1}\right)$ & \\
\hline Angular resolution ${ }^{\mathrm{a}}$........ & & $6.4 \times 5.9$ & \\
\hline Linear resolution ${ }^{\mathrm{a}}$. & & $100 \times 91 \mathrm{pc}$ & \\
\hline $\operatorname{rms~noise}^{\mathrm{a}} \ldots$ & & $\begin{array}{c}0.7 \mathrm{mJy} \mathrm{beam}^{-1} \\
11 \mathrm{~K}\end{array}$ & \\
\hline
\end{tabular}

${ }^{\text {a }}$ Based on ROBUST data cube; see text. 


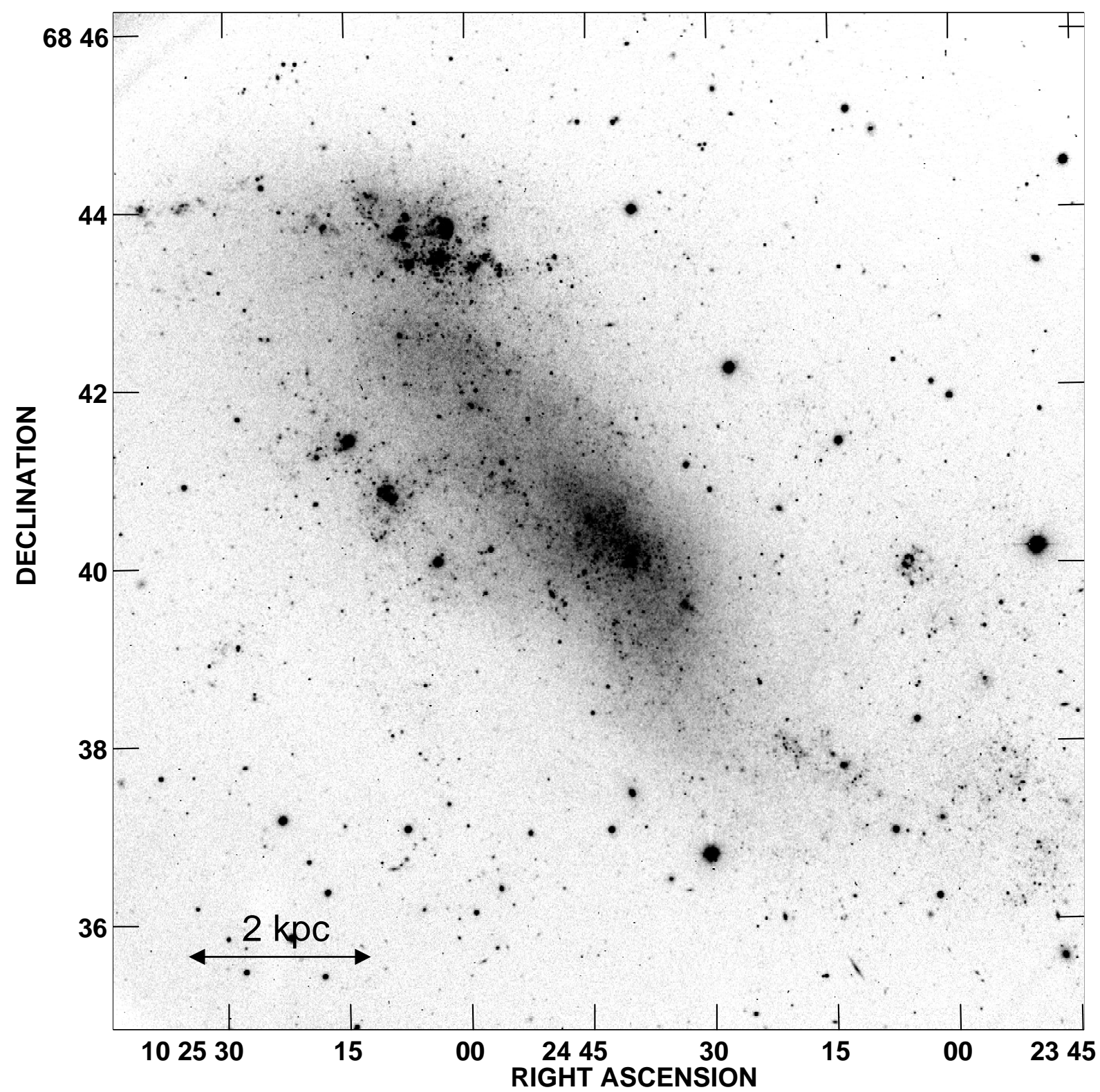

FIG. 1. $-R$-band CCD image of IC 2574 obtained with the Calar Alto $2.2 \mathrm{~m}$ telescope

smoothed map was then tested at the $2 \sigma$ level; if a pixel fell below this level, the counterpart in the cube was blanked. After that, the remaining peaks were inspected. Emission that was present in three consecutive channels was considered to be real, while all other remaining spikes were considered to be noise and blanked. The final result was named the master cube. This master cube can be used as a mask to blank the natural and ROBUST data cubes. This method ensures that the same regions are included when cubes are inspected at different resolutions and with different signal-to-noise ratios.

\subsection{Optical Observations}

IC 2574 was observed with the $2.2 \mathrm{~m}$ telescope of the Calar Alto Observatory ${ }^{4}$ on 1999 January 25 and 26 in

${ }^{4}$ The Calar Alto Observatory is operated by the Max-Planck-Institut für Astronomie (Heidelberg) jointly with the Spanish Comisión Nacional de Astronomía.
Johnson $R$-band (20 minutes) as well as in $\mathrm{H} \alpha$ (45 minutes). A $2048 \times 2048$ pixel CCD was used leading to 0 ".5 $\mathrm{pixel}^{-1}$. The seeing was around 1".3. A focal reducer (CAFOS) allowed for a field of view of radius $8^{\prime}$ to be imaged. The usual calibration steps were followed using the IRAF package $^{5}$. Each exposure was corrected for zero offset (bias) and was flat-fielded using skyflats. After that, frames were inspected for bad pixels and shifted to get them aligned. Lastly, the exposures were combined to eliminate cosmicray hits and to create a final image. As it turned out, conditions were not photometric during the observation, hence no calibration using standard stars was performed. In order to get an approximate calibration for IC 2574, values were taken from the literature (MCR94). The deep $R$-band image

${ }^{5}$ IRAF is distributed by National Optical Astronomy Observatories, which are operated by the Association of Universities for Research in Astronomy, Inc., under cooperative agreement with the National Science Foundation. 


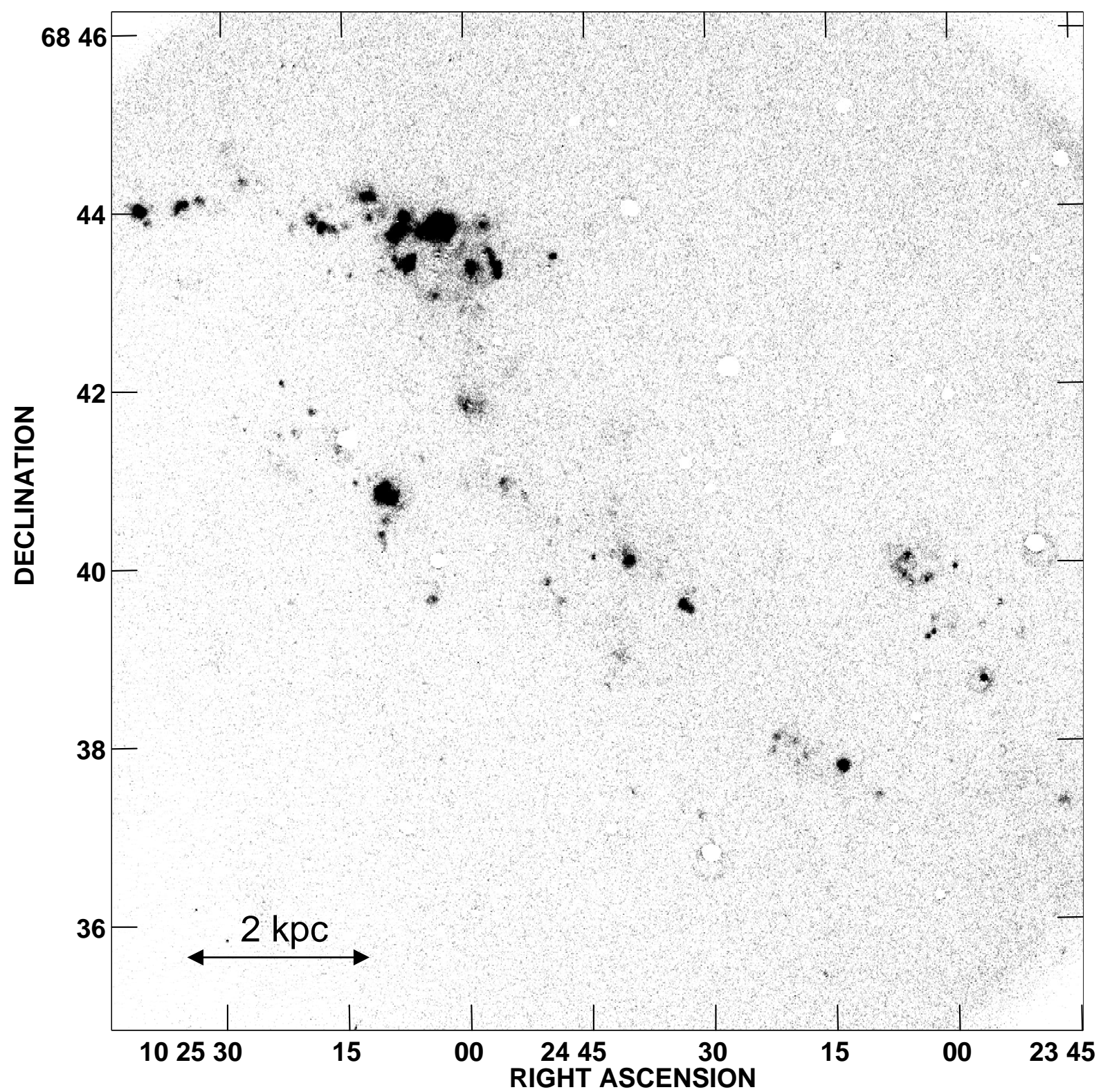

FIG. 2. Continuum-subtracted $\mathrm{H} \alpha$ image of IC 2574 obtained with the Calar Alto $2.2 \mathrm{~m}$ telescope. The total exposure time is $45 \mathrm{minutes}$. The brightest stars not fully subtracted by removing the continuum were blanked.

was used for the continuum subtraction of the $\mathrm{H} \alpha$ map. The continuum level was determined by simply scaling the continuum image relative to the line plus continuum image such that the foreground stars disappeared when the two images were subtracted. For the calibration we relied on the $\mathrm{H} \alpha$ fluxes published by MH94). These authors identify 289 $\mathrm{H}$ II regions in IC 2574 (for a thorough discussion on the $\mathrm{H} \alpha$ emission and its calibration see the Appendix in MH94). Figures 1 and 2 show our $R$-band and the $\mathrm{H} \alpha$ image, respectively.

Coordinates for the maps were obtained using the task KOORDS within the KARMA software package (for a description of KARMA see $\S 4.1$ ). We used as input an image from the Digital Sky Survey. Using 14 stars found in our CCD image, it was possible to determine the coordinate system to an accuracy of better than one arcsecond, more than adequate for our needs.

\section{H I STUDIES}

\subsection{Global H I Properties}

The channel maps obtained by the robust weighted cube are presented in Figure 3. As can be seen, most traces of contamination by Galactic emission were successfully removed. To save space, only every other channel over the frequency range where line emission is present is shown. The heliocentric radial velocities (in $\mathrm{km} \mathrm{s}^{-1}$ ) are plotted in the upper right-hand corner of each plane. The beam size $\left(6 " 4 \times 5^{\prime \prime} .9\right)$ is indicated in the top left-hand panel (note that the beam size is so small that it cannot be properly reproduced in our maps). Many holes and shell-like structures are visible in the channel maps; these features will be the main topic of this paper.

Obtaining the $\mathrm{H}$ I flux of each channel and hence the global H I profile of IC 2574 is not straightforward. As first 


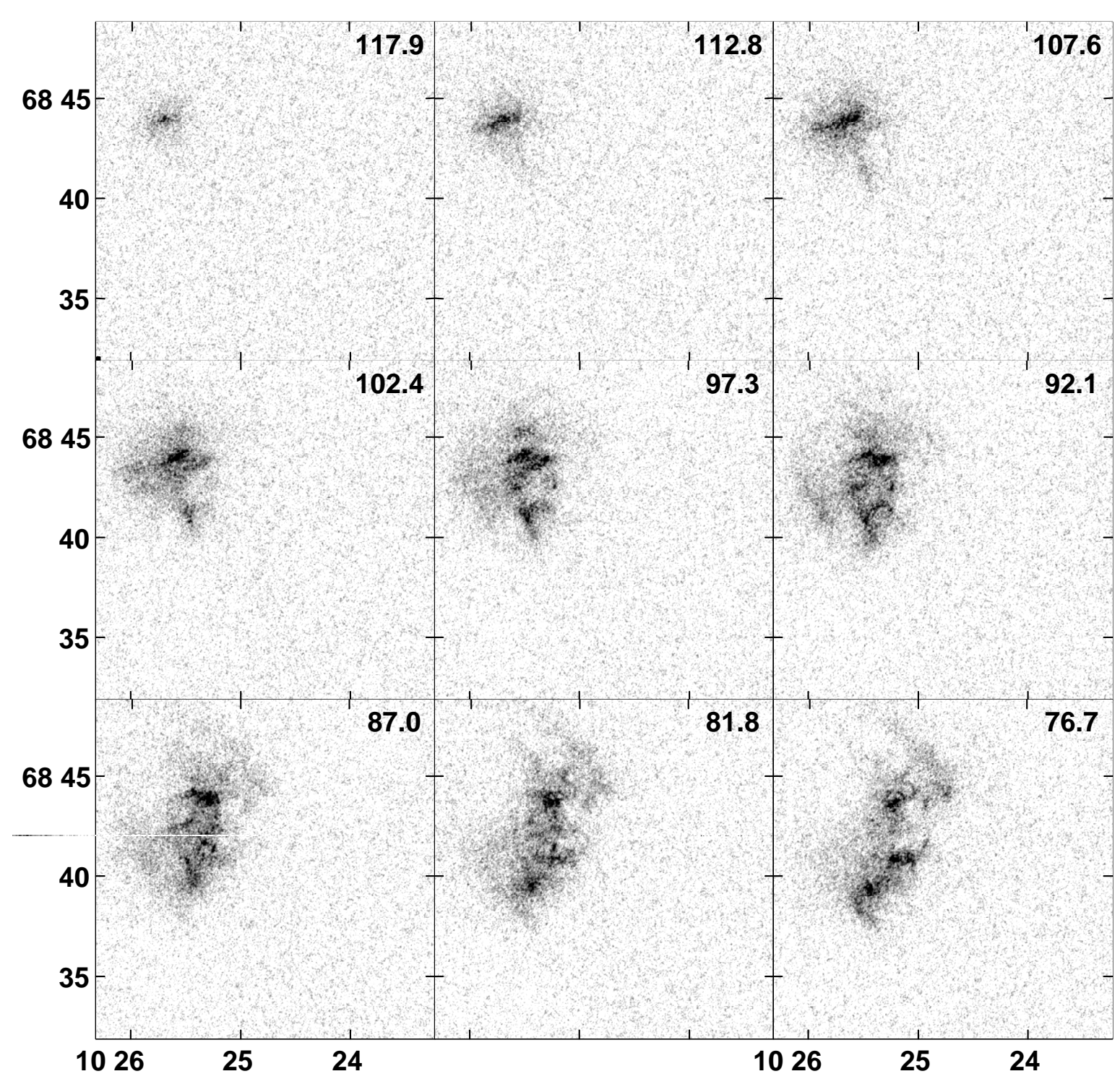

FIG. 3.-Mosaic of every second channel map of IC 2574 in which line emission was detected. The velocity of each channel in $\mathrm{km} \mathrm{s}^{-1}$ is plotted in the right-hand corner. Gray scale ranges from 0 (white) to $6 \mathrm{mJy} \mathrm{beam}^{-1}$ (black). Note that the beam size of 6"4 $\times$ 5".9 (indicated in the box of the top left-hand panel) is smaller than the resolution of this plot. Coordinates are with respect to B1950.0 equinox.

pointed out by Jörsäter \& van Moorsel (1995), fully cleaned maps do not exist and any cleaned map consists of the sum of two maps: one containing the restored clean components and the other the residual map. In the former, the unit is jansky per clean beam area, and in the latter jansky per dirty beam area. Usually, fluxes are determined on a cleaned map, assuming that the clean beam is the correct one for the entire map. In reality, the flux is calculated correctly only for the cleaned fraction of the map; for the residual map the flux is overestimated when the dirty beam area is bigger than the clean beam area (which is usually the case). This becomes a real problem for extended objects, especially in the case of combined-array data, like for our VLA data set. For a full discussion on this topic, see Jörsäter \& van Moorsel (1995). Following their prescription, the real flux of a channel map is given by:

$$
G=\frac{D \times C}{D-R}
$$

where $C$ is the cleaned flux and $R$ and $D$ are the "erroneous" residual flux and the "erroneous" dirty flux over the same area of a channel map, respectively. We followed this procedure for every single channel to obtain the global $\mathrm{H}$ I profile as presented in Figure 4. Without this correction, we would have overestimated the $\mathrm{H}$ i flux by a factor of about two!

Figure 4 shows the flux- and primary-beam corrected fluxes for every channel of IC 2574 (open squares) plotted together with the single-dish profile published by Rots (1980) and the WSRT data by MCR94 (the arrows indicate the velocity range for which the data points were interpolated because of contamination by galactic emission). From our data, we derive a total integrated $\mathrm{H}$ I flux of 373 $\mathrm{Jy} \mathrm{km} \mathrm{s}^{-1}$, which should be compared with the value of 399 Jy km s${ }^{-1}$ obtained by Rots (1980), who used the Greenbank 300 foot telescope to map IC 2574. This is excellent agreement, given the uncertainties of removing Galactic emission and systematic errors in the absolute calibration. 


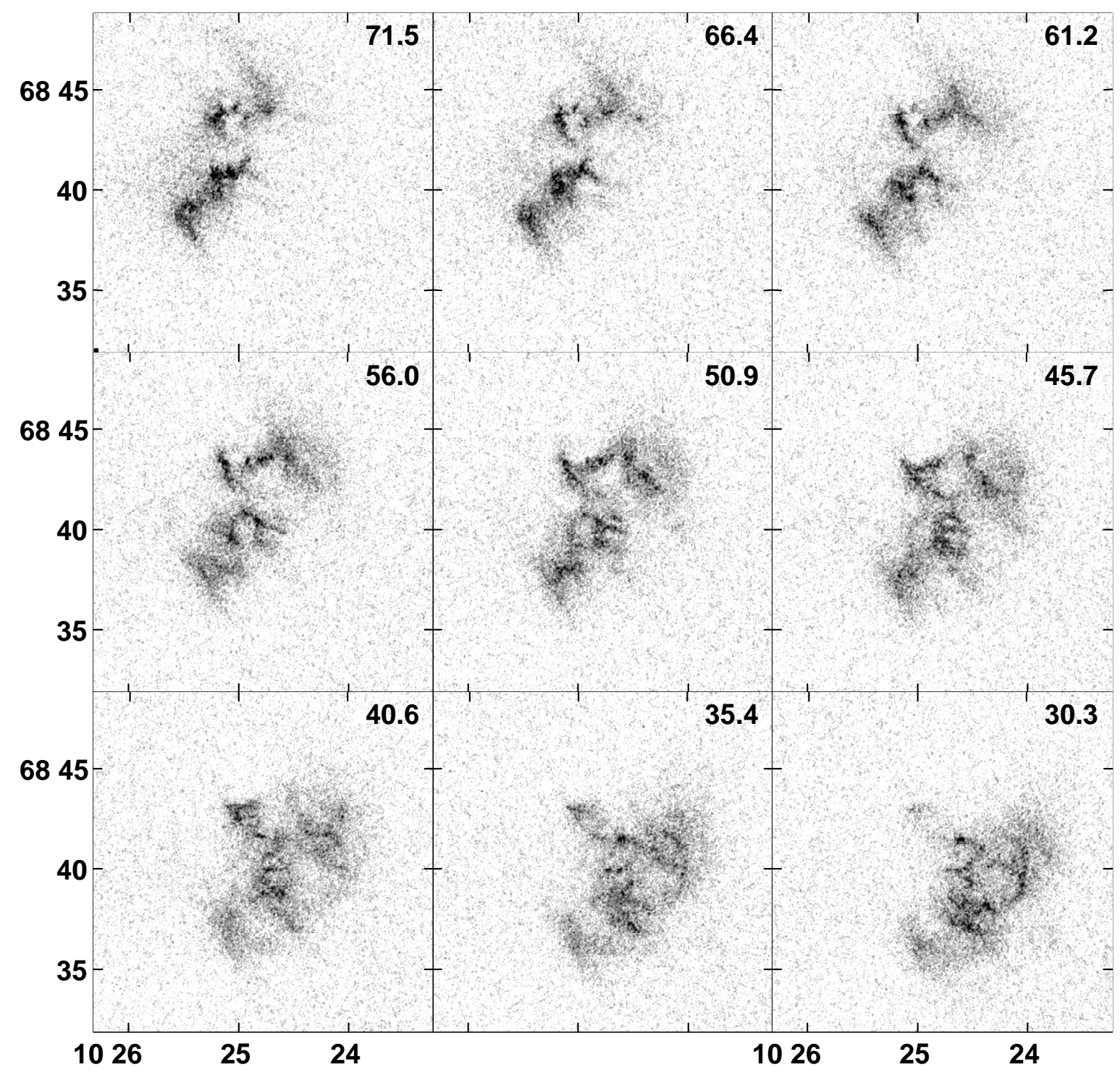

FIG. 3.-Continued

This also means that we miss hardly any $\mathrm{H}$ i flux due to missing short spacings. Other values obtained by interferometric observations are: $440 \pm 50 \mathrm{Jy} \mathrm{km} \mathrm{s}{ }^{-1}$ found by Seielstad \& Wright (1973), using the Owens Valley Radio Interferometer and $286 \mathrm{Jy} \mathrm{km} \mathrm{s}{ }^{-1}$ obtained by MCR94 from their Westerbork observations. As can be seen from Figure 4, the new VLA observations recover substantially more flux than the WSRT data. Adopting a distance of 3.2 Mpc, our flux translates to a total neutral hydrogen mass of $M_{\mathrm{HI}}=(9.0 \pm 0.1) \times 10^{8} M_{\odot}$ for IC 2574 .

\subsection{Global Characteristics}

Figure 5 shows the H I surface density map of IC 2574 obtained by adding all channels containing $\mathrm{H}$ I emission of the robust cube, after blanking them with the master cube. Note that the beam size is so small that it can hardly be properly reproduced. Note also that, although many holelike structures and cavities are visible, specific features turn out to be less prominent in the total surface brightness map than in the channel maps. This is related to the fact that the galaxy is highly inclined, leading to overlapping of shell-like structures along the line-of-sight in the integrated map. For a more complete discussion on that topic, see $\S 4.1$.

Figure 6 shows the velocity field of IC 2574, which was calculated in the usual manner by taking the first moment. Since the high-resolution velocity field is dominated by small-scale motions within the ISM, the natural weighted cube was convolved to 30" (as indicated by the beam size) to put more emphasis on the global characteristics of the velocity field. The underlying gray scale is a linear representation of the $\mathrm{H}$ I surface brightness map at full resolution. Although the overall velocity field shows the typical behavior for a dwarf galaxy (solid-body rotation in the center, flattening of the rotation curve toward the very edge of the galaxy), the general rotation is obviously disturbed by noncircular motions (e.g., note the prominent band of lower velocities to the northeast of the center of the galaxy, parallel to its major axis). These deviations are linked to holes in the neutral interstellar medium.

Finally, Figure 7 shows the second moment map, or velocity dispersion, of IC 2574. Although some regions near shells reach values as high as $15 \mathrm{~km} \mathrm{~s}^{-1}$, the overall velocity 


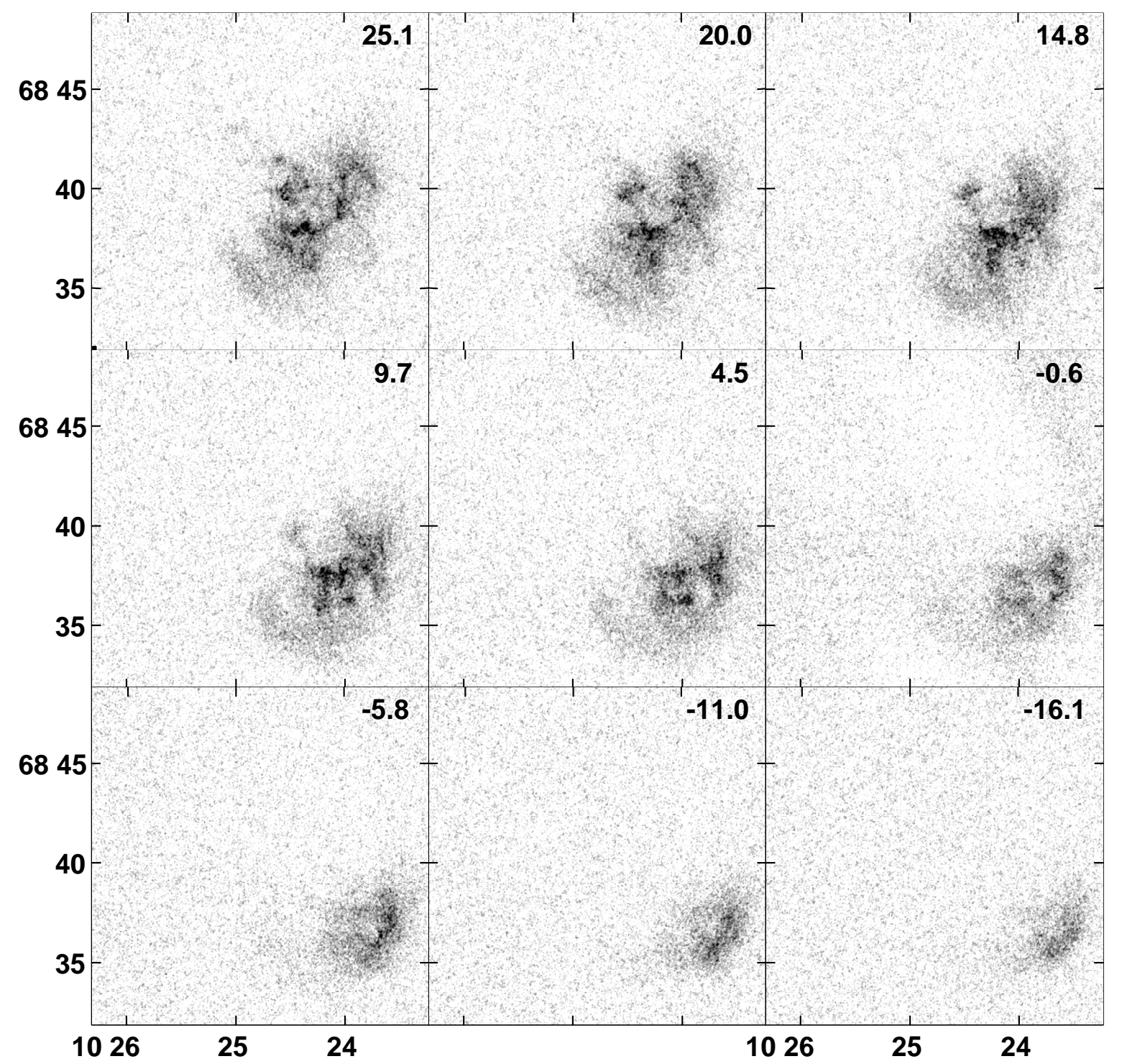

FIG. 3.-Continued

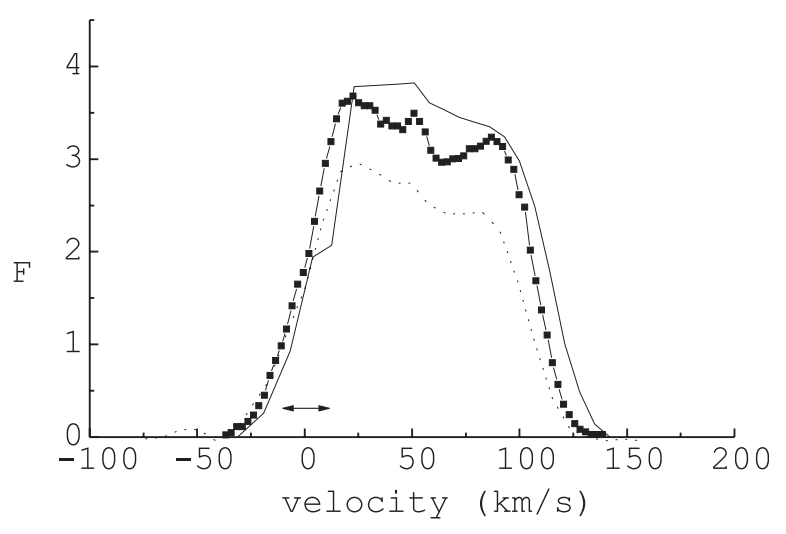

FIG. 4.-Global H I profile for IC 2574 (squares) obtained by integrating the channel maps over the area of the source after subtraction of the continuum background. The data have been flux corrected (see text) and are corrected for primary-beam attenuation. The flux $F$ is given in Jy. The channel spacing is $2.58 \mathrm{~km} \mathrm{~s}^{-1}$. For comparison, the profiles published by Rots (1980) (solid line) and MCR94 (dotted line) are also plotted. The arrow indicates the velocity range affected by contamination due to galactic emission. dispersion in the unperturbed parts is much lower. Using $\mathrm{H}$ I profiles, we derive the velocity dispersion in the disk by looking at quiescent regions. Several lines of sight were averaged to give a $1 \sigma$ velocity dispersion of $7 \pm 1 \mathrm{~km} \mathrm{~s}^{-1}$ (similar to what is found in Ho II). This shows that our velocity resolution of $2.5 \mathrm{~km} \mathrm{~s}^{-1}$ (FWHM) was quite sufficient to resolve the lines of neutral hydrogen.

In this paper, we focus on the small-scale structure of the ISM in IC 2574. For our analysis, however, we need to adopt values for the orientation of the object, which are usually derived on the basis of a kinematical analysis (rotation curve fitting). A full analysis of the dynamics of IC 2574 based on our observations, however, is beyond the scope of this paper. Luckily, an adequate analysis has already been published (see the paper by MCR94). In the course of their study they derive a systemic velocity of $58 \pm 7 \mathrm{~km} \mathrm{~s}^{-1}$, adopt an inclination of $75^{\circ} \pm 7^{\circ}$ and a position angle of $52^{\circ}$. Depending on their dynamical model, they find that $70 \%-90 \%$ of the total mass of the galaxy is in the form of dark matter. Following the same procedure as 


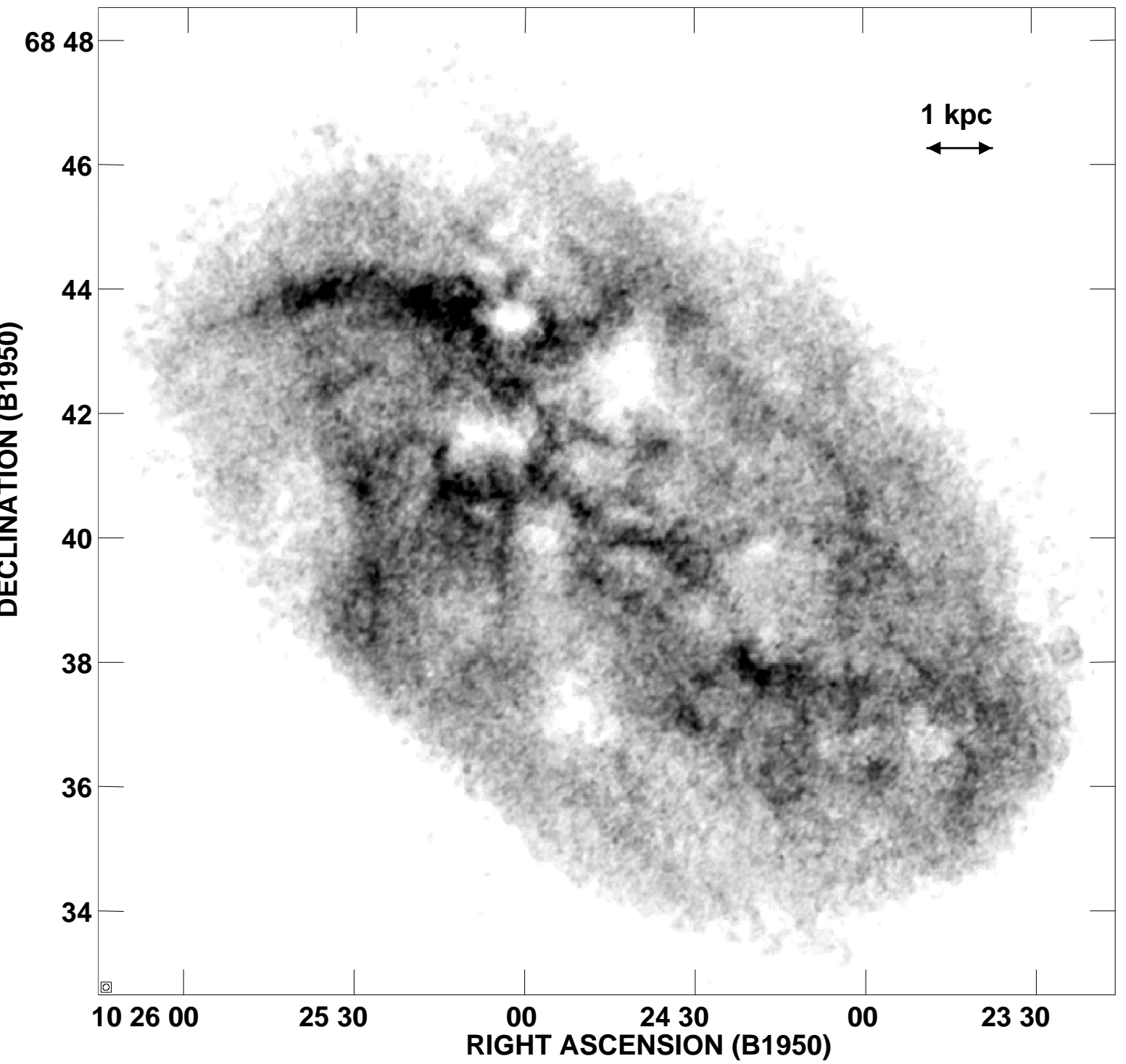

FIG. 5.- - H I surface-brightness map of IC 2574 . The gray scale is a linear representation of the $\mathrm{H}$ i surface brightness $\left(0-2406 \mathrm{~K}^{\mathrm{km} \mathrm{s}}{ }^{-1}\right)$. The beam size $(6.4 \times 5.9)$ is indicated in the lower left.

performed for the dwarf galaxy II Zw 33 (Walter et al. 1997), we carried through a first analysis of our data that confirmed the results by MCR94 regarding the rotation curve and the global orientation of IC 2574. For ease of comparison we therefore adopted their values throughout this paper.

\subsection{The Scale Height of the $\mathrm{H}$ I Disk}

The scale height $h$ of the H I layer of IC 2574 plays an important role in the determination of the properties of the holes, e.g., when translating the observed $\mathrm{H}$ i surface density into an $\mathrm{H}$ I volume density. Here $h$ is defined as the $1 \sigma$ value for a Gaussian distribution of the $\mathrm{H}$ I layer. Recent studies have shown that in general dwarf galaxies have thicker $\mathrm{H}$ I disks than more massive spiral galaxies. Their aspect ratio tends to be of the order of 10:1 rather than 100:1, e.g., Ho II: $h=625$ pc (PWBR92) or NGC 5023: $h=460$ pc (Bottema, Shostak, \& van der Kruit 1986; see also StaveleySmith et al. 1992). In the case of IC 2574 there are also indications that the $\mathrm{H} \mathrm{I}$ is distributed in a thicker disk. MCR94 already noticed that, looking at the apparent axial ratio of the integrated $\mathrm{H}$ I map and assuming a real inclina- tion of $75^{\circ}$, the $\mathrm{H}$ I must be distributed in a somewhat thicker disk. Moreover, the fact that we observe, as we will argue below, spherically expanding shells with diameters of up to almost $1 \mathrm{kpc}$ implies that the extent of the $\mathrm{H}$ I layer must be of the same order of magnitude. Hence, the scale height of the $\mathrm{H}$ I gas should be of order $350-400 \mathrm{pc}$.

An approximate value for the scale height can be obtained fairly simply, as shown by PWBR92. We follow their approach here. The scale height is proportional to the velocity dispersion of the gas and inversely proportional to the square root of the mass density in the disk (Kellmann 1972; van der Kruit 1981). For a density distribution that can be described as $\rho(z, R)=\rho(0, R) \operatorname{sech}\left(z / z_{0}\right) 2, z_{0}$ is given by

$$
z_{0}(R)=\frac{\sigma_{\mathrm{gas}}}{\sqrt{2 \pi G \rho(0, R)}}
$$

where $R$ is the galactocentric distance, $\sigma_{\text {gas }}$ the velocity dispersion of the gas in quiescent regions of the galaxy $(\S 3.2)$, and $\rho(R)$ the total volume density (gas, stars, and dark matter) of the galaxy in the plane. However, since $\operatorname{sech}\left(z / z_{0}\right) \approx \exp \left(-z^{2} / z_{0}^{2}\right) 2$ (to within $5 \%$ in the region of 


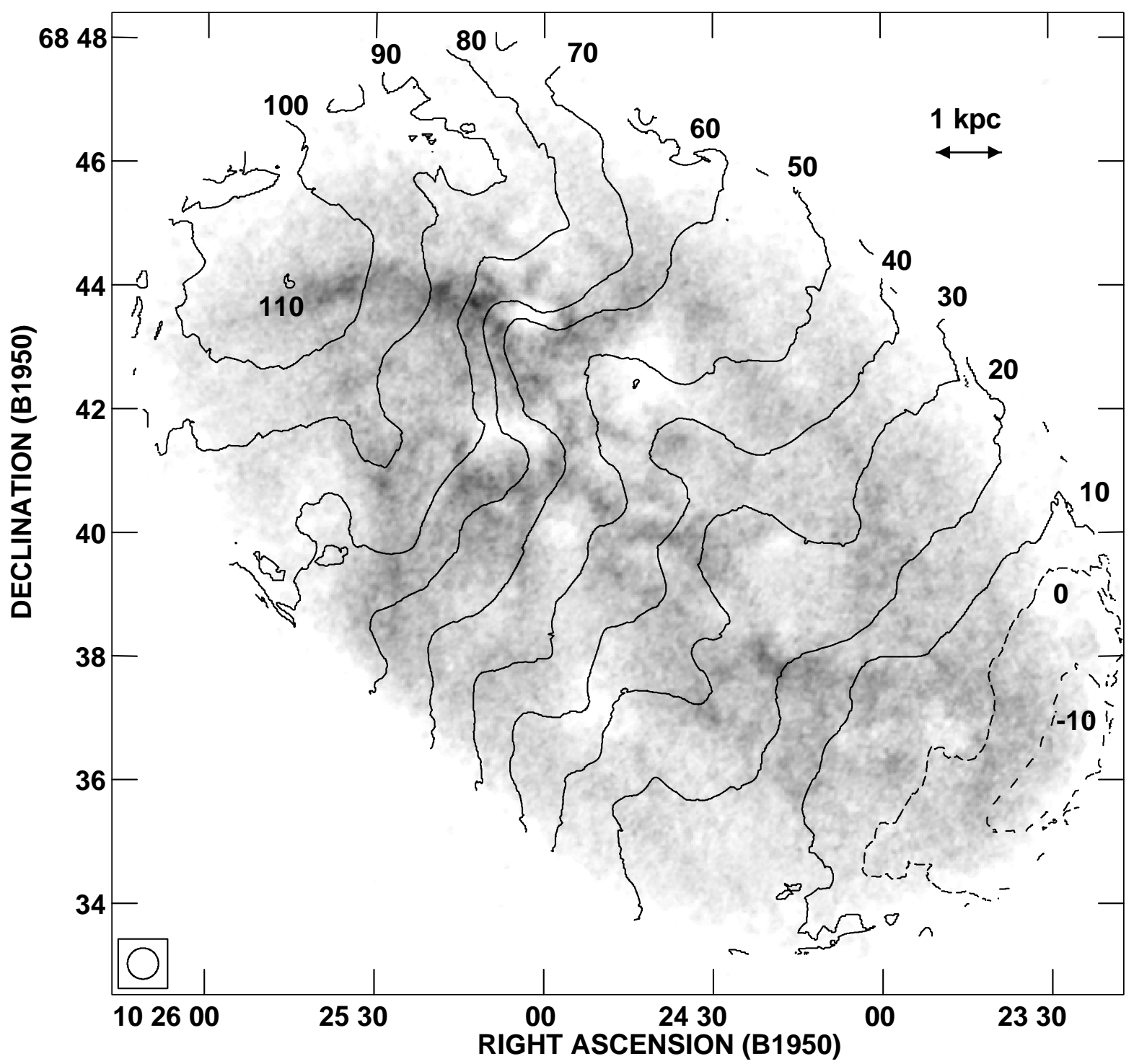

FIG. 6.-Isovelocity contours of IC 2574 of the $30^{\prime \prime}$ convolved cube. The velocities are given in $\mathrm{km} \mathrm{s}^{-1}$. The gray-scale image is a linear representation of the $\mathrm{H}$ I surface-brightness map at full resolution. A circular $30^{\prime \prime}$ beam is plotted in the lower left hand corner.

interest) we can also assume a Gaussian distribution in $z$. The $1 \sigma$ scale height $h$ is then defined as $h=z_{0} / 2^{-1 / 2}$. To calculate the average density of IC 2574 in the plane we assume that the total mass of the galaxy can be estimated from the last measured point $\left(R_{\max }\right)$ of the observed rotation curve (MCR94) and that this is distributed over a spherical volume of radius $R_{\max }$. MCR94 derive a rotation curve with $v\left(R_{\max }\right)=66 \mathrm{~km} \mathrm{~s}^{-1}$ at $R_{\max }=8.0 \mathrm{kpc}$. This corresponds to a total dynamical mass of $8.3 \times 10^{9} M_{\odot}$. Distributing this mass over a spherical volume with radius $R_{\max }$ gives an average value for $\rho$ of $0.0039 M_{\odot} \mathrm{pc}^{-3}$. Since this is only the mean density of the galaxy and the density near the plane is certainly higher, we take twice this derived value (see PWBR92 for a justification) to get a first approximation to the real volume density $\rho(R)$ near the center. Using this approach, we find $\rho(R)=0.008 M_{\odot} \mathrm{pc}^{-3}$.

Substituting the observed velocity dispersion of $7 \mathrm{~km} \mathrm{~s}^{-1}$ (see $\S 3.2$ ) in the equation given above, we derive a scale height of $h=345 \mathrm{pc}$ for IC 2574 . Note that this value, given all the uncertainties in the derivation, is in good agreement with the value derived based on the sizes of the largest holes $(h=350-400 \mathrm{pc})$. We therefore adopted a scale height of $h=350 \mathrm{pc}$ for the H I layer of IC 2574 .

We can now calculate the volume density of the $\mathrm{H}$ I from the deprojected (face-on) H I surface density. The surface density profile was obtained by elliptically averaging the flux- and primary-beam corrected total H I map (assuming an inclination of $75^{\circ}$ ). Again, this is correct to first order only; as in the case of a thick $\mathrm{H}$ I distribution, the deprojection of the observed projected $\mathrm{H}$ I distribution is not trivial. We then calculated the volume density $n_{\mathrm{H}}(R)$ of the $\mathrm{H} \mathrm{I}$ in the plane of the disk using

$$
N_{\mathrm{HI}}(R)=\int_{-\infty}^{+\infty} n_{\mathrm{HI}}(R) \exp \left(\frac{-z^{2}}{2 h^{2}}\right) d z=\sqrt{2 \pi} h n_{\mathrm{H} \mathrm{I}}(R)
$$

\section{H I HOLES IN IC 2574}

\subsection{H I-Hole Catalog}

We conducted a search for $\mathrm{H}$ I structures in IC 2574 using the tasks KVIEW, KPVSLICE, and KSHELL that are 
6848

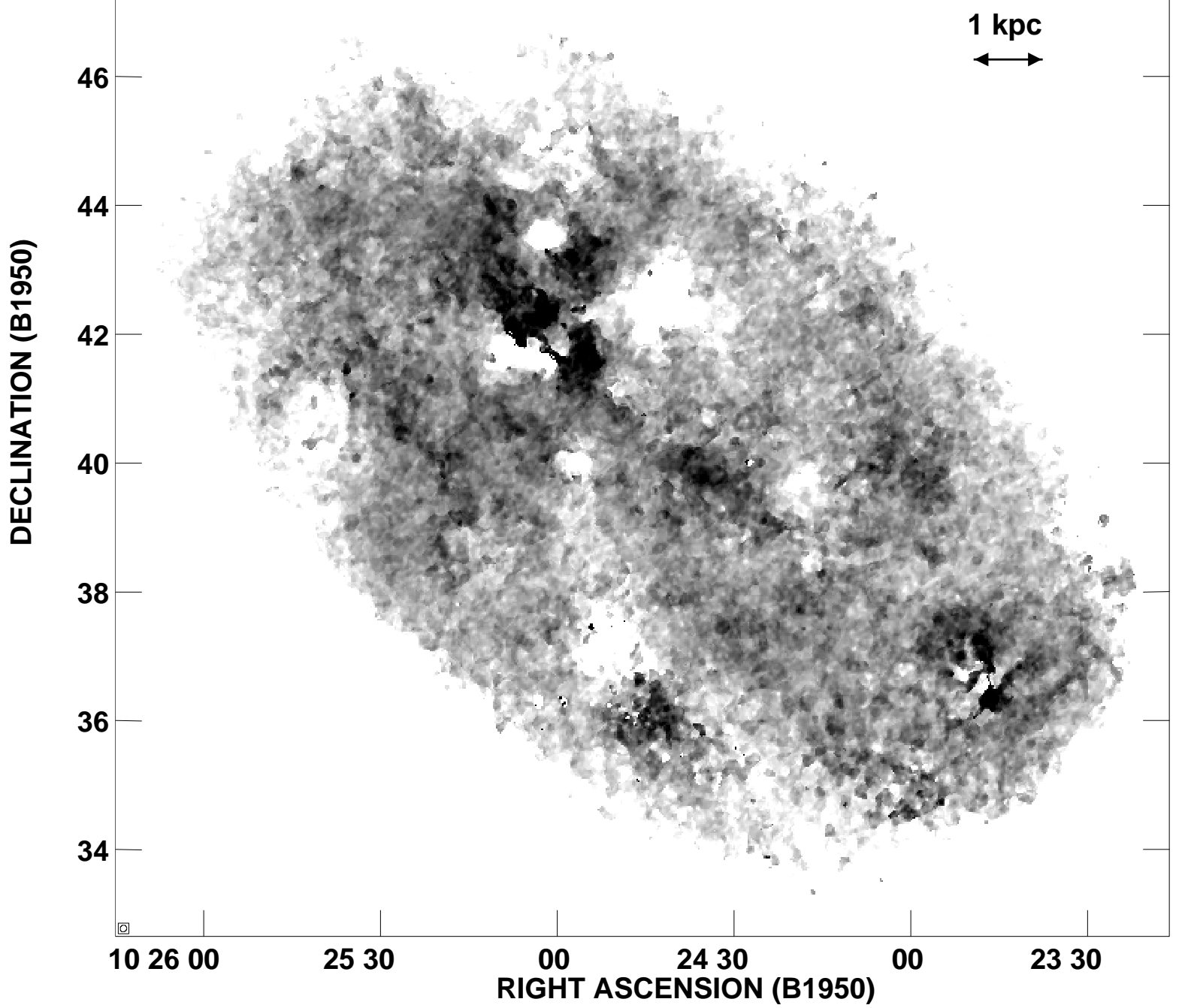

FIG. 7.-Velocity-dispersion (second moment) map of IC 2574 . The gray scale is a linear representation of the velocity dispersion ranging between $4 \mathrm{~km}$ $\mathrm{s}^{-1}$ (white) and $13 \mathrm{~km} \mathrm{~s}^{-1}$ (black). The beam size $\left(6^{\prime \prime} .4 \times 5^{\prime \prime} .9\right)$ is indicated in the lower left.

built into the recently developed KARMA ${ }^{6}$ visualization software package. KVIEW offers many different ways to look at a movie of a data cube, whereas KPVSLICE produces real-time position velocity $(\mathrm{pV})$ cuts through a cube in any orientation, which tremendously facilitates the search for and identification of holelike structures. Finally, KSHELL was used to make radius-velocity plots of some of the more prominent shells and holes.

First, the ROBUST cube was searched for the smaller holes in IC 2574. In order to be sensitive to more extended structures and therefore bigger holes, we smoothed our ROBUST cube to $11^{\prime \prime}, 15^{\prime \prime}$, and $30^{\prime \prime}$, respectively, and inspected each of them. For each H I hole in IC 2574 we determined its position, size, and expansion velocity. Each author independently produced a list of holes to reduce

\footnotetext{
${ }^{6}$ The KARMA visualization software package was developed by Richard Gooch of the Australia Telescope National Facility (ATNF).
}

personal bias, based on some simple criteria regarding the determination of the center, size, expansion velocity, and systemic velocity of the holes. After that, the two lists were compared and merged. There was an overlap of about $75 \%$ in terms of well-defined holes, which lends some credibility to the identification process. We reexamined the remaining structures and, erring on the side of caution, ended up with a catalog of 48 holes. Although this list cannot be free from personal bias, the method closely follows that employed in the case of M31 and Ho II, which should make a comparison between those objects and IC 2574 valid.

It is interesting, at this point, to reflect on the reality of the features. Do they really constitute large-scale, coherent structures or are we tricked by nature into seeing coherence in what is basically a turbulent, perhaps fractal, medium? Mac Low et al. (1998) recently produced sets of model data cubes, similar to those obtained via radio (H I) or optical $(\mathrm{H} \alpha)$ observations (i.e., position, position, and velocity). Using the same techniques as explained above, we analyzed 
a cube of uniform, isotropic, isothermal, supersonic, superAlfvénic, decaying turbulence. The simulations assume that the gas is optically thin and emits with a strength directly proportional to its density. The model corresponds to a weakly magnetized ISM. Single cuts through the data cube show features remarkably similar to the ones seen in the $\mathrm{H} \mathrm{I}$ data. However, when focusing on a potential feature and investigating its morphology in position-position or position-velocity space, no coherent structure emerges. This leads us to believe that shells due to random (or at least turbulent) processes make up a small fraction of the holes we classified. Instead, it is more likely that the $\mathrm{H}$ I holes and shells are caused by mechanisms that act on large scales, such as stellar winds and (multiple) supernovae.

Following Brinks \& Bajaja (1986), we distinguished between three different types of holes based on their appearance in the $\mathrm{pV}$ diagrams. A type 1 hole corresponds to a total blowout (i.e., we observe neither the receding nor the approaching side of the shell). In that case, it is impossible to determine the expansion velocity and hence the kine-
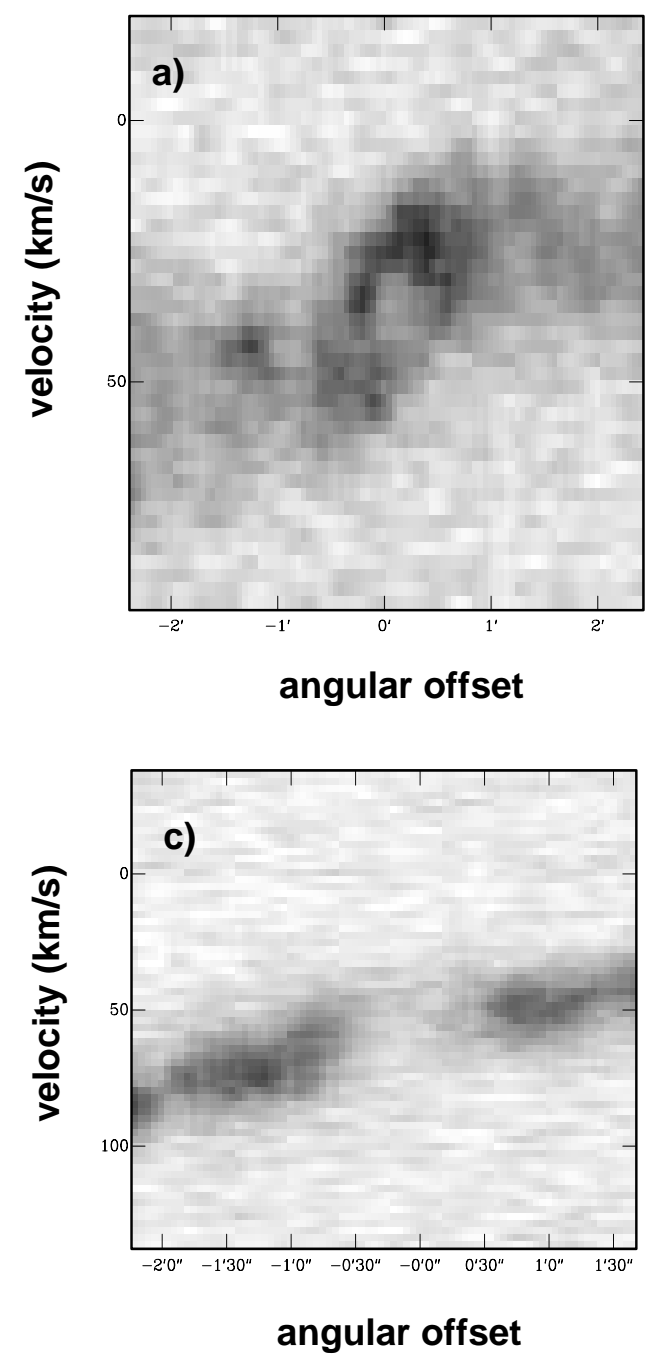

matics of the hole. Figure $8 c$ shows a $\mathrm{pV}$ diagram of a typical type 1 hole (our hole number 30) using KPVSLICE.

A type 2 hole corresponds to a hole that is offset with respect to the plane of the galaxy. In that case, we witness a deformation in the $\mathrm{pV}$ diagram toward the plane of the galaxy and a blowout to the opposite side (see Silich et al. 1996 for numerical simulations of such a case - their case C). The amount of deformation yields the expansion velocity of the shell toward the midplane. The part of the shell that points away from the plane is usually not visible because of a lack of sensitivity. Figure $8 d$ shows a $\mathrm{pV}$ diagram of a typical type 2 hole (our hole number 33 ).

Type 3 is the "classic hole," corresponding to a more or less complete expanding shell. The signature in $\mathrm{pV}$ space is that of an elliptical hole. The expansion velocity is equal to half the difference between the velocity of the approaching and receding sides. The systemic velocity of the hole is defined by its center. Figure $8 a$ shows a $\mathrm{pV}$ diagram of a typical example of a type 3 hole (our hole number 21 ). Figure $8 b$ shows the same hole, but analyzed with the task
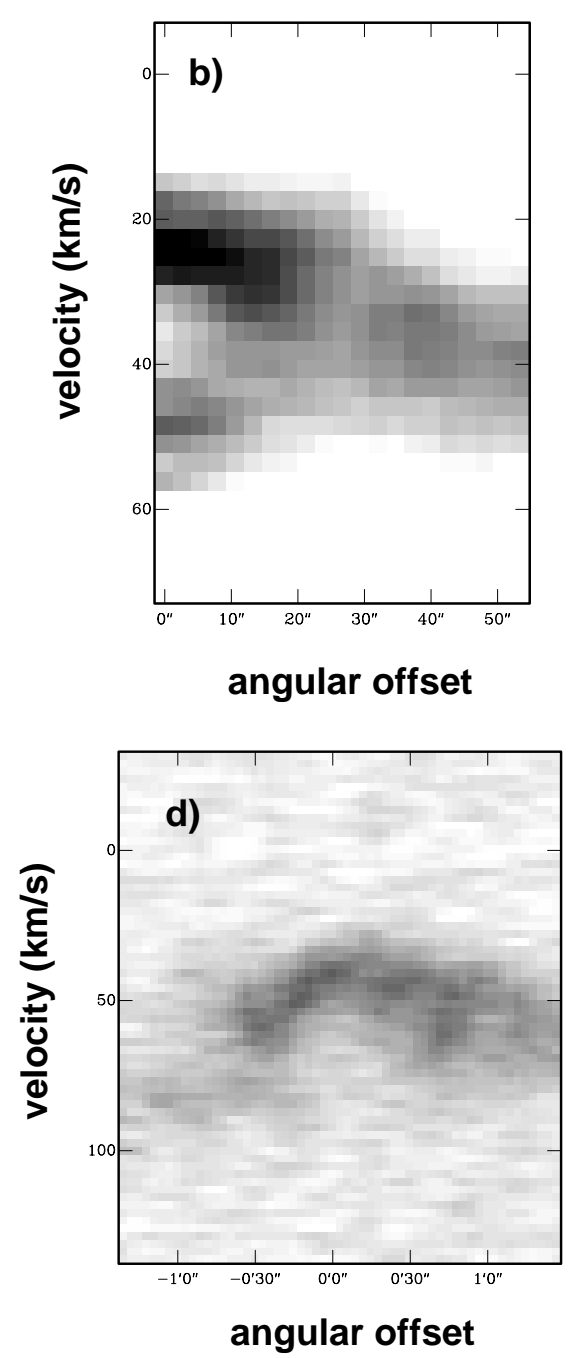

FIG. 8.-Typical examples of position-velocity diagrams. (a) $\mathrm{pV}$ diagram of a type 3 hole (hole number 21 ). (b) $\mathrm{rV}$ diagram of the same hole. (c) $\mathrm{pV}$ diagram of a type 1 hole (number 30 ). And (d) pV diagram of a type 2 hole (number 33 ). 
KSHELL. In this figure, the horizontal axis corresponds to radius $r$ (distance from the center of the hole) and the intensity corresponds to the average $\mathrm{H}$ I density within an annulus with radius $r$ around the center of the hole. The vertical axis is again velocity. Since we are looking at a radius-velocity diagram, we will call such a representation an $\mathrm{rV}$ diagram in the following. For an ideal shell, a half ellipse should be visible in which the diameter in the $v$ direction is twice the expansion velocity and its dimension in the $r$ direction the radius of the shell. The advantage of looking at a near circular $\mathrm{H}$ I shell with KSHELL lies in a substantial increase in signal-to-noise ratio as one averages over many pixels, whereas in a $\mathrm{pV}$ diagram one merely cuts through an $\mathrm{H}$ i structure.

Note that in the case of a type 1 hole, the absence of detectable emission corresponding to the front and back of a shell causes a hole in $\mathrm{rV}$ space. For obvious reasons, a type 2 hole does not show a prominent signature in an $\mathrm{rV}$ diagram either.

As was already mentioned earlier, the holes were found to be much more prominent in the channel maps than in the integrated $\mathrm{H}$ I map. This is due to the high inclination of IC 2574, which leads to several $\mathrm{H}$ I shells at different locations within the disk and possibly different velocities overlapping along the line-of-sight, as is the case in M31. The total H I surface brightness map was therefore of little use for the identification of a hole. Note that, in the case of Ho II, only the integrated map was used because of the much lower inclination of the galaxy. This implies that the approach to search for holes needs to be adapted to the orientation of the galaxy.

Figure 9 shows a plot of the distribution of the $48 \mathrm{H} \mathrm{I}$ holes detected with confidence in IC 2574. The gray-scale map is a linear representation of the total $\mathrm{H}$ I surface brightness. Some of the holes are clearly visible in the total H I map but the majority of holes does not show up at all and can only be detected in the channel maps or $\mathrm{pV}$ diagrams.

The final list of the detected $\mathrm{H}$ I holes is given in Table 3. The properties listed are as follows:

1. Number of the hole (increasing with increasing right ascension).

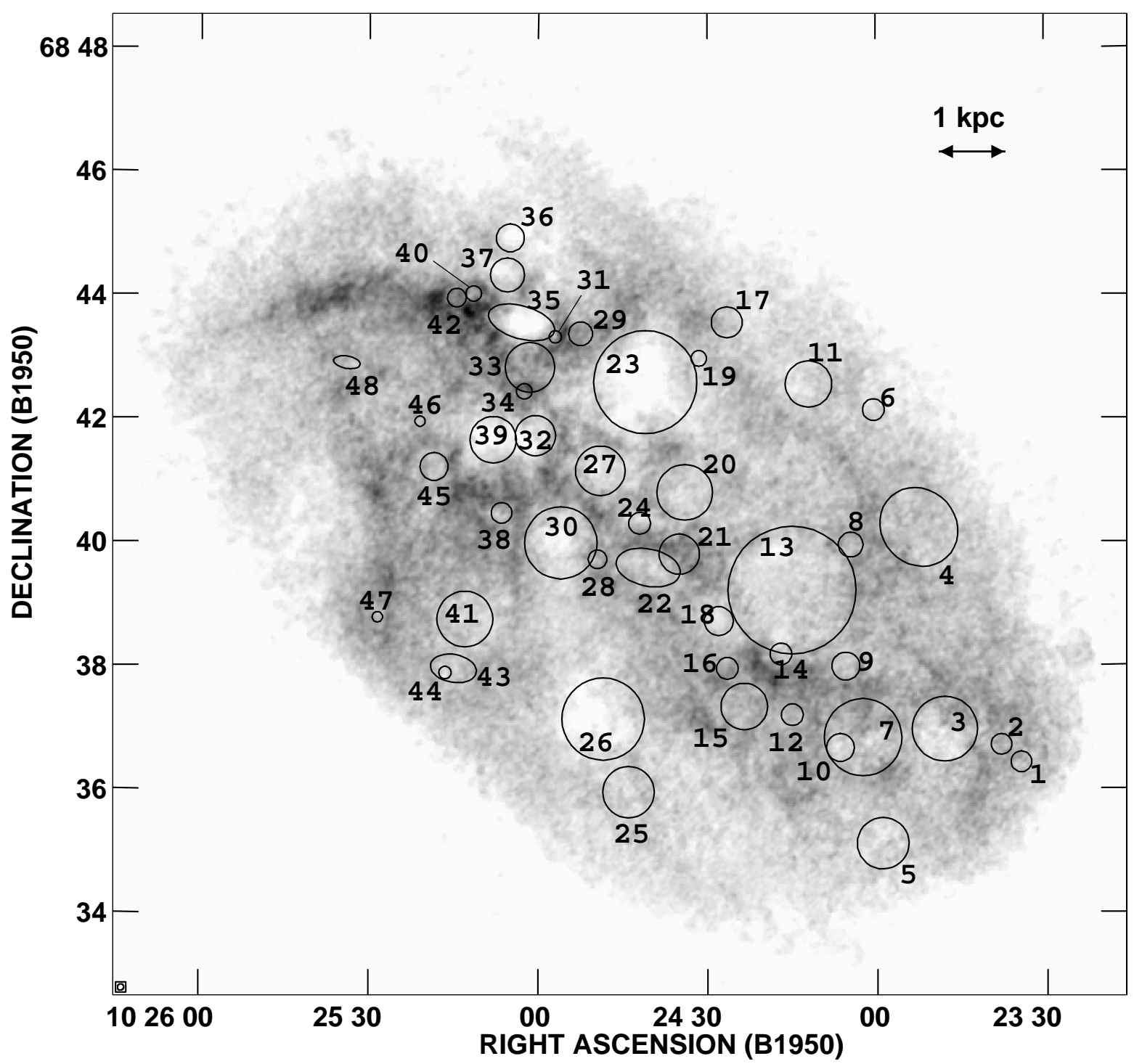

FIG. 9.- Location of the H I holes in IC 2574 (with numbers). The gray-scale map is a linear representation of the H I surface-brightness map. For the largest holes (13,23, and 26), see text. 
TABLE 3

ObSERved Properties of the H i Holes

\begin{tabular}{|c|c|c|c|c|c|c|c|}
\hline $\begin{array}{l}\text { Number } \\
\text { (1) }\end{array}$ & $\begin{array}{c}\alpha(1950.0) \\
(2)\end{array}$ & $\begin{array}{c}\delta(1950.0) \\
(3)\end{array}$ & $\begin{array}{c}v_{\text {hel }}\left(\mathrm{km} \mathrm{s}^{-1}\right) \\
(4)\end{array}$ & $\begin{array}{l}d(\mathrm{pc}) \\
(5)\end{array}$ & $\begin{array}{c}v_{\exp }\left(\mathrm{km} \mathrm{s}^{-1}\right) \\
(6)\end{array}$ & $\begin{array}{c}n_{\mathrm{HI}}\left(\mathrm{cm}^{-3}\right) \\
\quad(7)\end{array}$ & $\begin{array}{c}\text { Type } \\
(8)\end{array}$ \\
\hline $1 \ldots \ldots \ldots$ & 102334.5 & 683626 & -11 & 310 & 8 & 0.13 & 3 \\
\hline $2 \ldots \ldots \ldots$ & 102338.0 & 683643 & -1 & 310 & 5 & 0.13 & 3 \\
\hline $3 \ldots \ldots$ & 102348.0 & 683658 & 5 & 976 & 17 & 0.15 & 3 \\
\hline $4 \ldots \ldots \ldots$ & 102352.5 & 684014 & 28 & $1240 \times 1116,42^{\circ}$ & 14 & 0.05 & 2 \\
\hline $5 \ldots \ldots \ldots$ & 102359.0 & 683407 & -1 & 775 & 9 & 0.1 & 3 \\
\hline $6 \ldots \ldots \ldots$ & 102400.5 & 684208 & 53 & 325 & 8 & 0.04 & 3 \\
\hline $7 \ldots \ldots \ldots$ & 102402.5 & 683650 & 2 & 1162 & - & 0.16 & 1 \\
\hline $8 \ldots \ldots \ldots$ & 102404.6 & 683957 & 33 & 372 & 15 & 0.08 & 3 \\
\hline $9 \ldots \ldots \ldots$ & 102405.5 & 683759 & 17 & 418 & 7 & 0.16 & 3 \\
\hline $10 \ldots \ldots$ & 102406.5 & 683640 & 7 & 418 & - & 0.15 & 1 \\
\hline $11 \ldots \ldots$ & 102412.0 & 684233 & 43 & 697 & 8 & 0.04 & 3 \\
\hline $12 \ldots \ldots$ & 102415.0 & 683712 & 15 & 325 & - & 0.16 & 1 \\
\hline $13 \ldots \ldots$ & 102415.0 & 683913 & 25 & 1922 & - & 0.16 & 1 \\
\hline $14 \ldots \ldots$ & 102417.0 & 683811 & 20 & 325 & - & 0.17 & 1 \\
\hline $15 \ldots \ldots$ & 102423.5 & 683720 & 25 & 697 & 6 & 0.16 & 3 \\
\hline $16 \ldots \ldots$ & 102426.5 & 683757 & 25 & 325 & 8 & 0.16 & 3 \\
\hline $17 \ldots \ldots$ & 102426.5 & 684333 & 53 & 465 & 13 & 0.08 & 3 \\
\hline $18 \ldots \ldots$ & 102428.0 & 683843 & 28 & 434 & 11 & 0.17 & 3 \\
\hline $19 \ldots \ldots$ & 102431.5 & 684258 & 36 & 232 & 8 & 0.07 & 3 \\
\hline $20 \ldots \ldots$ & 102442.0 & 684018 & 33 & 837 & - & 0.16 & 1 \\
\hline $21 \ldots \ldots$ & 102435.0 & 683948 & 38 & 604 & 18 & 0.17 & 3 \\
\hline $22 \ldots \ldots$ & 102440.5 & 683935 & 38 & $976 \times 558,78^{\circ}$ & 13 & 0.17 & 3 \\
\hline $23 \ldots \ldots$ & 102441.0 & 684235 & 46 & 1550 & - & 0.11 & 1 \\
\hline $24 \ldots \ldots$ & 102442.0 & 684018 & 46 & 325 & - & 0.17 & 1 \\
\hline $25 \ldots \ldots$ & 102444.0 & 683557 & 25 & 775 & 13 & 0.05 & 3 \\
\hline $26 \ldots \ldots$ & 102448.5 & 683708 & 33 & 1240 & - & 0.07 & 1 \\
\hline $27 \ldots \ldots$ & 102449.0 & 684109 & 48 & 744 & - & 0.17 & 1 \\
\hline $28 \ldots \ldots$ & 102449.5 & 683943 & 53 & 279 & 6 & 0.17 & 3 \\
\hline $29 \ldots \ldots$ & 102452.5 & 684322 & 56 & 356 & 10 & 0.09 & 3 \\
\hline $30 \ldots \ldots$ & 102456.0 & 683959 & 53 & 1085 & - & 0.17 & 1 \\
\hline $31 \ldots \ldots$ & 102457.0 & 684319 & 51 & 186 & - & 0.12 & 1 \\
\hline $32 \ldots \ldots$ & 102500.5 & 684143 & 61 & 604 & 24 & 0.17 & 3 \\
\hline $33 \ldots \ldots$ & 102501.5 & 684249 & 66 & 744 & 23 & 0.15 & 2 \\
\hline $34 \ldots \ldots$ & 102502.5 & 684226 & 48 & 232 & 9 & 0.16 & 3 \\
\hline $35 \ldots \ldots$ & 102503.0 & 684333 & 64 & $1023 \times 496,74^{\circ}$ & 25 & 0.13 & 2 \\
\hline $36 \ldots \ldots$ & 102505.0 & 684455 & 82 & 418 & 10 & 0.07 & 3 \\
\hline $37 \ldots \ldots$ & 102505.5 & 684419 & 77 & 511 & - & 0.07 & 1 \\
\hline $38 \ldots \ldots$ & 102506.5 & 684028 & 69 & 310 & - & 0.15 & 1 \\
\hline $39 \ldots \ldots$ & 102508.0 & 684139 & 64 & 697 & - & 0.17 & 1 \\
\hline $40 \ldots \ldots$ & 102511.5 & 684401 & 77 & 232 & 10 & 0.12 & 3 \\
\hline $41 \ldots \ldots$ & 102513.0 & 683845 & 59 & 837 & - & 0.06 & 1 \\
\hline $42 \ldots \ldots$ & 102514.5 & 684357 & 77 & 279 & 13 & 0.13 & 2 \\
\hline $43 \ldots \ldots$ & 102515.0 & 683757 & 56 & $697 \times 418,80^{\circ}$ & - & 0.05 & 2 \\
\hline $44 \ldots \ldots$ & 102516.5 & 683753 & 56 & 186 & 8 & 0.05 & 3 \\
\hline $45 \ldots \ldots$ & 102518.5 & 684113 & 87 & 418 & 7 & 0.16 & 3 \\
\hline $46 \ldots \ldots$ & 102521.8 & 684157 & 87 & 155 & 4 & 0.16 & 3 \\
\hline $47 \ldots \ldots$ & 102528.5 & 684113 & 71 & 155 & 8 & 0.05 & 3 \\
\hline $48 \ldots \ldots$ & 102534.0 & 684254 & 92 & $403 \times 186,80^{\circ}$ & 8 & 0.15 & 2 \\
\hline
\end{tabular}

NotE.- Units of right ascension are hours, minutes, and seconds, and units of declination are degrees, arcminutes, and arcseconds.

2. Position of the center of the hole in right ascension and declination, respectively (B1950.0 coordinates). Typical errors are $3^{\prime \prime}$.

3. Heliocentric velocity $v_{\text {hel }}$ of the hole defined by the channel in which the hole is largest or most prominent. The accuracy of $v_{\text {hel }}$ is about $2.5 \mathrm{~km} \mathrm{~s}^{-1}$ (the velocity resolution of our observations).

4. Diameter $d$ of the hole in kpc. Note that most of the holes are circular. In the case of elliptical holes, the diameter along the major axis, along the minor axis and the position angle $\left(2 \times b_{\text {maj }}, 2 \times b_{\text {min }}\right.$, P.A.) is given (but see $\left.\S 4.2\right)$. The accuracy of $d$ is around 95 pc (the FWHM of our synthe- sized beam).

5. Expansion velocity $v_{\exp }$ of the hole in $\mathrm{km} \mathrm{s}^{-1}$. Note that, in the case of a type 1 hole, no expansion velocity can be measured. Typical errors are about $2 \mathrm{~km} \mathrm{~s}^{-1}$.

6. Estimated $\mathrm{H}$ I volume density $n_{\mathrm{HI}}$ (in $M_{\odot} \mathrm{pc}^{-3}$ ) of the region where the hole is situated before the hole was created. We calculated $n_{\mathrm{HI}}$ based on the $\mathrm{H}$ I surface density, as described in $\S 3.3$. Given the uncertainties in the deprojection and the assumptions that went into the derivation of the mass density within the disk, we estimate a relative error in the value of $n_{\mathrm{H}}$ to be of order $30 \%$.

7. The type of the hole (1-3), as defined in $\S 4.1$. 
Table 4 summarizes the physical properties derived from Table 3. The properties listed are as follows:

1. Number of the hole.

2. Diameter $d$ of the hole. For elliptical holes, the diameter was defined using the geometric mean

$$
d=\sqrt{2 b_{\mathrm{maj}} \times 2 b_{\mathrm{min}}} .
$$

3. Expansion velocity $v_{\exp }$ of the hole in $\mathrm{km} \mathrm{s}^{-1}$. Same as column (6) in Table 3.

4. Kinematic age of the hole in units of megayears. This age determination assumes that over its entire lifetime the hole was expanding with $v_{\text {exp }}$. Hence:

$$
t=\frac{(d / 2)}{v_{\exp }} .
$$

The accuracy of the kinetic age is typically $20 \%$. Because of the assumption of a constant expansion velocity, the kinematic age is an upper limit.

5. Indicative $\mathrm{H}_{\mathrm{I}}$ mass $M_{\mathrm{HI}}$ in units of $10^{4} M_{\odot}$. This is the mass that filled the observed hole under the following assumptions: (a) the hole is spherical, (b) the gas was distributed uniformly in the galaxy, and (c) the hole is completely empty now. Note that if one can assume that only a minor

TABLE 4

\begin{tabular}{|c|c|c|c|c|c|}
\hline Number & $d(\mathrm{pc})$ & $\begin{array}{c}v_{\exp } \\
\left(\mathrm{km} \mathrm{s}^{-1}\right)\end{array}$ & $\begin{array}{c}\text { Age } t \\
\left(10^{6} \mathrm{yr}\right)\end{array}$ & $\begin{array}{l}\text { Mass } M_{\mathrm{H} \mathrm{I}} \\
\left(10^{4} M_{\odot}\right)\end{array}$ & $\begin{array}{l}\text { Energy } E_{\mathrm{E}} \\
\left(10^{50} \mathrm{ergs}\right)\end{array}$ \\
\hline $1 \ldots \ldots \ldots$ & 310 & 8 & 19.6 & 4.9 & 6.8 \\
\hline $2 \ldots \ldots \ldots$ & 310 & 5 & 31.3 & 4.9 & 3.5 \\
\hline $3 \ldots \ldots \ldots$ & 976.5 & 17 & 29.0 & 178.4 & 817.9 \\
\hline $4 \ldots \ldots \ldots$ & 1178 & 14 & 42.5 & 104.4 & 326.9 \\
\hline $5 \ldots \ldots \ldots$ & 775 & 9 & 43.5 & 59.5 & 103.7 \\
\hline $6 \ldots \ldots \ldots$ & 325.5 & 8 & 20.6 & 1.8 & 2.1 \\
\hline $7 \ldots \ldots$ & 1162.5 & $\ldots$ & $\ldots$ & 321.1 & $\ldots$ \\
\hline $8 \ldots \ldots$. & 372 & 15 & 12.2 & 5.2 & 16.7 \\
\hline $9 \ldots \ldots$ & 418.5 & 7 & 30.2 & 15.0 & 18.1 \\
\hline $10 \ldots \ldots$ & 418.5 & $\ldots$ & $\ldots$ & 14.0 & $\ldots$ \\
\hline $11 \ldots \ldots$ & 697.5 & 8 & 44.1 & 17.3 & 22.7 \\
\hline $12 \ldots \ldots$ & 325.5 & $\ldots$ & $\ldots$ & 7.0 & $\ldots$ \\
\hline $13 \ldots \ldots$ & 1922 & $\ldots$ & $\ldots$ & 1451.2 & $\ldots$ \\
\hline $14 \ldots \ldots$ & 325.5 & $\ldots$ & $\ldots$ & 7.5 & $\ldots$ \\
\hline $15 \ldots \ldots$ & 697.5 & 6 & 58.7 & 69.4 & 71.6 \\
\hline $16 \ldots \ldots$ & 325.5 & 8 & 20.6 & 7.0 & 9.9 \\
\hline $17 \ldots \ldots$ & 465 & 13 & 18.1 & 10.3 & 27.4 \\
\hline $18 \ldots \ldots$ & 434 & 11 & 19.9 & 17.8 & 40.7 \\
\hline $19 \ldots \ldots$ & 232.5 & 8 & 14.7 & 1.1 & 1.4 \\
\hline $20 \ldots \ldots$ & 837 & $\ldots$ & $\ldots$ & 119.9 & $\ldots$ \\
\hline $21 \ldots \ldots$ & 604.5 & 18 & 17.0 & 48.0 & 228.3 \\
\hline $22 \ldots \ldots$ & 744 & 13 & 28.9 & 89.4 & 276.7 \\
\hline $23 \ldots \ldots$ & 1550 & $\ldots$ & $\ldots$ & 523.3 & $\ldots$ \\
\hline $24 \ldots \ldots$ & 325.5 & $\ldots$ & $\ldots$ & 7.5 & $\ldots$ \\
\hline $25 \ldots \ldots$ & 775 & 13 & 30.1 & 29.7 & 79.8 \\
\hline $26 \ldots \ldots$ & 1240 & $\ldots$ & $\ldots$ & 170.5 & $\ldots$ \\
\hline $27 \ldots \ldots$ & 744 & $\ldots$ & $\ldots$ & 89.4 & $\ldots$ \\
\hline $28 \ldots \ldots$ & 279 & 6 & 23.5 & 4.7 & 4.4 \\
\hline $29 \ldots \ldots$ & 356.5 & 10 & 18.0 & 5.2 & 9.5 \\
\hline $30 \ldots \ldots$ & 1085 & $\ldots$ & $\ldots$ & 277.4 & $\ldots$ \\
\hline $31 \ldots \ldots$ & 186 & $\ldots$ & $\ldots$ & 1.0 & $\ldots$ \\
\hline $32 \ldots \ldots$ & 604.5 & 24 & 12.7 & 48.0 & 341.5 \\
\hline $33 \ldots \ldots$ & 744 & 23 & 16.3 & 78.9 & 534.6 \\
\hline $34 \ldots \ldots$ & 232.5 & 9 & 13.1 & 2.6 & 4.1 \\
\hline $35 \ldots \ldots$ & 713 & 25 & 14.3 & 60.2 & 420 \\
\hline $36 \ldots \ldots$ & 418.5 & 10 & 21.1 & 6.6 & 11.8 \\
\hline $37 \ldots \ldots$ & 511.5 & $\ldots$ & $\ldots$ & 12.0 & $\ldots$ \\
\hline $38 \ldots \ldots$ & 310 & $\ldots$ & $\ldots$ & 5.7 & $\ldots$ \\
\hline $39 \ldots \ldots$ & 697.5 & $\ldots$ & $\ldots$ & 73.7 & $\ldots$ \\
\hline $40 \ldots \ldots$ & 232.5 & 10 & 11.7 & 1.9 & 3.4 \\
\hline $41 \ldots \ldots$ & 837 & $\ldots$ & $\ldots$ & 44.9 & $\ldots$ \\
\hline $42 \ldots \ldots$ & 279 & 13 & 10.8 & 3.6 & 9.6 \\
\hline $43 \ldots \ldots$ & 542.5 & $\ldots$ & $\ldots$ & 10.2 & $\ldots$ \\
\hline $44 \ldots \ldots$ & 186 & 8 & 11.7 & 0.4 & 0.5 \\
\hline $45 \ldots \ldots$ & 418.5 & 7 & 30.2 & 15.0 & 18.1 \\
\hline $46 \ldots \ldots$ & 155 & 4 & 19.6 & 0.8 & 0.4 \\
\hline $47 \ldots \ldots$ & 155 & 8 & 9.8 & 0.2 & 0.3 \\
\hline $48 \ldots \ldots$ & 279 & 8 & 17.6 & 4.2 & 5.7 \\
\hline
\end{tabular}

DerIVEd Properties OF THE H I Holes 
fraction of the surrounding $\mathrm{H} \mathrm{I}$ is ionized, this mass corresponds also to the total $\mathrm{H}$ I mass on the rim of the hole. $M_{\mathrm{HI}}$ was calculated using

$$
M_{\mathrm{HI}}=\frac{4}{3} \pi(d / 2)^{3} n_{\mathrm{HI}}(R),
$$

where $n(R)$ is the volume density of the gas at the galactocentric distance $R$ (col. [7] in Table 3). To correct this value for the amount of primordial helium, a factor of 1.33 has to be applied. The relative error is, as $d$ enters to the third power, of order $30 \%$. Note that in the case of the largest holes, this value is more likely to be an upper limit since $n_{\mathrm{HI}}$ decreases with increasing distance $z$ from the plane.

6. The initial total energy $E_{\mathrm{E}}$ that was released by the supernova explosions that created the hole (in units of $10^{50}$ ergs). Note that the kinetic energy of the expanding shell is about $1 \%$ to $10 \%$ of $E_{\mathrm{E}}$ (depending on the ambient density; see Chevalier 1974). To determine $E_{\mathrm{E}}$, we used the equation by Chevalier (1974) who derived a fit to numerical results of an expanding shell created by a single explosion (see also McCray \& Snow 1979). Expressing $E_{\mathrm{E}}$ in terms of observable quantities $d, v_{\exp }$ and $n_{0}$, he obtained

$$
E_{\mathrm{E}}=5.3 \times 10^{43} n_{0}^{1.12}(d / 2)^{3.12} v_{\exp }^{1.4} \text { ergs },
$$

where $v_{\exp }$ is the expansion velocity in $\mathrm{km} \mathrm{s}^{-1}, n_{0}$ the density of the ambient medium in particles per cubic centimeter, and $d$ is the diameter of the shell in parsecs. In our calculations we replaced $n_{0}$ by $n_{\mathrm{H}}$, as was done, e.g., for M31 and Ho II. This is not strictly correct, because we are ignoring contributions by $\mathrm{He}$ and heavier elements. To correct $E_{\mathrm{E}}$ for this contribution, all values should be multiplied by a factor of $\approx 1.5$. In addition, the derived $\mathrm{H}$ I densities are average values for a given galactocentric distance, which introduces another source of uncertainty. Note that the numerical coefficient in Chevalier's equation depends on the radiative energy loss rate and thus on the heavy element abundance in the expanding shell. Not only is this abundance different for various galaxies, but it decreases with galactic radius, both for our own Galaxy and for external galaxies and by more than an order of magnitude (e.g., Heiles 1979). The last phase in the expansion of a shell is reached when, after the shell has continued to collect more of the ambient medium, it will slow down to velocities comparable to the random motions of the ISM (around $7 \mathrm{~km}$ $\mathrm{s}^{-1}$ ) and hence stop expanding. Most probably, the largest structures we observe are in this latter phase (see $\S 4.3$ ). From this brief discussion it should be clear that the derived energies $E_{\mathrm{E}}$ are only order of magnitude estimates.

\subsection{Notes on Single Holes}

The following holes deserve a more detailed description:

Hole 35.- This hole causes a major disturbance in the $\mathrm{pV}$ diagrams of IC 2574. It is one of the few elliptical holes, with dimensions of $1000 \times 500 \mathrm{pc}$. Although there is some circumstantial evidence that it is a superposition of two spherical holes, it was treated as one hole. As we will show in $\S 5$, the most prominent $\mathrm{H}$ II region in IC 2574 is located on the rim of this supergiant shell. The shell is filled with soft X-ray emission as observed with the ROSAT telescope. A detailed multiwavelength analysis (optical, radio, X-ray) of this particular supergiant shell has been presented elsewhere (Walter et al. 1998).
Hole 22.-Cataloged as elliptical in shape, this hole is actually a superposition of several holes giving the overall impression of an elliptical feature; we treated this as a single object because subdividing it into, say, three holes would have been arbitrary.

Holes 13, 23, and 26.-As can be seen from the channel maps (Fig. 3), some holes are not clear-cut cases in terms of the definition of a hole given in $\S 4.1$. They are possibly chance superpositions of old shells and fragments giving the impression of a hole in the integrated H I map (see also $\S 4.3)$. We therefore decided not to include these holes in the statistical analysis.

\subsection{Notes on Large-Scale Structures}

As can be seen from Table 3, only few shells larger than $\approx 1000 \mathrm{pc}$ have been cataloged in IC 2574 . It must be mentioned, however, that coherent features larger then $1 \mathrm{kpc}$ appear in the channel maps. Several of these can be identified in the channel maps (Fig. 3), e.g., between 45 and $70 \mathrm{~km}$ $\mathrm{s}^{-1}$. Note that these structures are related to the major disturbance in the velocity field due north of the center of the galaxy. Obviously, it is very difficult to catalog these features, because they lack the clear coherence of $\mathrm{H}$ i shells, both in position as well as in velocity. Moreover, because of their large size, their dynamics is not dominated by some leftover expansion, but instead by large-scale galactic rotation modified by the effects of nearby, smaller holes. The reason we wish to mention these large-scale features is because along some of the apparent arcs we find $\mathrm{H}$ II regions.

\section{THE H I-H $\alpha$ CONNECTION IN IC 2574}

\subsection{Global Correlations}

Until now, we have focussed on the structure and dynamics of the neutral ISM of IC 2574. In the following, we compare these results with our $\mathrm{H} \alpha$ observations, which reveal the sites of recent or ongoing SF in IC 2574. As noted earlier $(\S 1)$, the expanding $\mathrm{H}$ I structures are widely thought to result from recent star formation. They sweep up gas causing the volume density on the rim of the shell to increase until gravitational instabilities lead to molecular cloud formation and hence secondary SF (e.g., Palouš et al. 1990; Elmegreen 1994). Therefore, a comparison of the position of $\mathrm{H}$ I holes with $\mathrm{H}$ II regions should show some correlation, the $\mathrm{H} \alpha$ emission being predominantly found along the rims of larger holes, as evidence for propagating SF. In the case of the smaller, young holes one would expect some to be still filled with diffuse $\mathrm{H} \alpha$ emission coming from the ionized wind-blown cavities around the still present, young $\mathrm{O}$ and $\mathrm{B}$ stars.

Figure 10 shows an overlay of the $\mathrm{H} \alpha$ map with the $\mathrm{H}$ I holes we detected in our survey $(\S 4.1)$. The width of the rims of the $\mathrm{H}$ I holes is of course in reality larger then indicated in this plot. The subregions $\mathrm{A}-\mathrm{C}$ will be discussed in more detail in the following section. As can be seen from this overlay, there is a prominent correlation of ongoing SF with the rims of the large $\mathrm{H}$ I shells which is in general agreement with the picture painted above. There is a strong tendency for $\mathrm{H}$ II regions to be situated on the rims of the $\mathrm{H}$ I shells (for some very nice examples see holes 8 and 35 ) and only in a very few cases diffuse $\mathrm{H} \alpha$ emission does not 


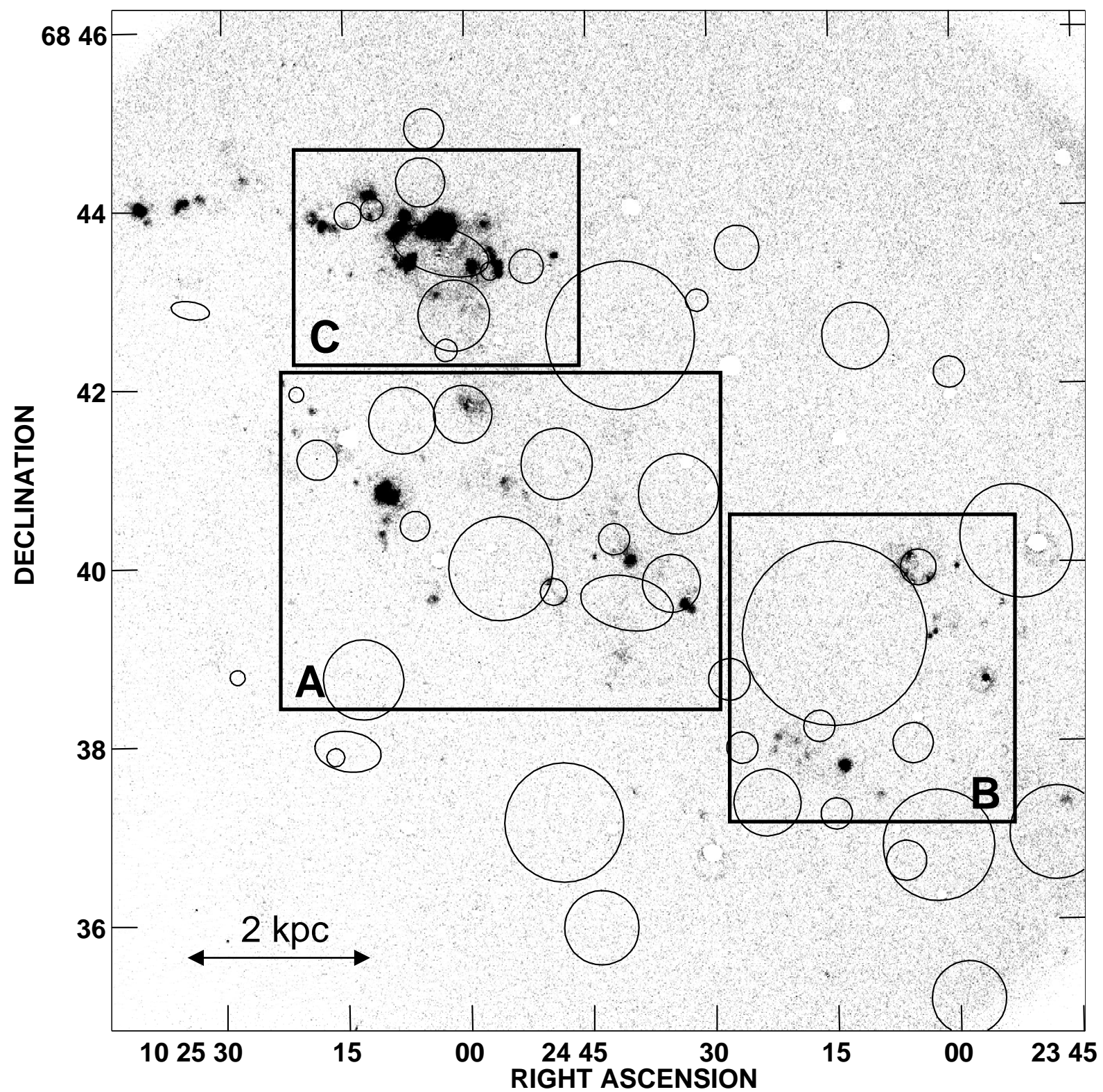

FIG. 10. - H $\alpha$ image of IC 2574 overlaid with the discovered holes in its neutral interstellar medium. Regions A, B, and C are enlarged in Figs. 12, 13, and 14, respectively. Note that almost all $\mathrm{H} \alpha$ emission in IC 2574 is located on the rims of $\mathrm{H}$ I shells (see text).

seem to be related to an $\mathrm{H}$ I structure. Note that in one case only (hole 32), diffuse $\mathrm{H} \alpha$ emission (partly) fills an $\mathrm{H}$ i hole.

Figure 11 presents an overlay of the $\mathrm{H} \alpha$ map with one contour of the $\mathrm{H}$ I surface brightness map (flux and primary beam corrected) plotted at $1.7 \times 10^{21}$ atoms $\mathrm{cm}^{-2}$. As can be seen from this overlay, $\mathrm{H} \alpha$ emission is globally correlated with a high $\mathrm{H}$ I surface brightness (for an exception in the very north see below). Kennicutt (1989) used the disk instability relation of Toomre (1964) to show that, for spirals, there is a threshold density for star formation. For gas densities above the threshold, the star formation rate (SFR) is consistent with a Schmidt law. Near the threshold, the correlation breaks down, and one sees bursts of SF. Much below the threshold the SFR is extremely low. Skillman (1987) has observationally determined this threshold for dwarf galaxies to be on the order of $1 \times 10^{21}$ atoms cm $\mathrm{cm}^{-2}$ (at a resolution of $500 \mathrm{pc}$ ), which is in general agreement with our results (see also below). For a detailed discussion on the models that describe the relationship between gas, stars and star formation in irregular galaxies, the reader is referred to the paper by Hunter, Elmegreen, \& Baker (1998).

\subsection{Analysis of Individual $\mathrm{H}$ II Regions}

Using our high-resolution H I data of IC 2574, we are now able to perform a much more detailed analysis and comparison of the properties of the $\mathrm{H}$ in regions with the $\mathrm{H} \mathrm{I}$ features that are related to them. When making overlays of the $\mathrm{H} \alpha$ map with the high-resolution cube we found that in virtually all cases, single $\mathrm{H}$ II regions are correlated with distinct $\mathrm{H}$ I structures like clumps or clouds. We defined 40 regions where $\mathrm{H} \alpha$ emission and $\mathrm{H}$ I clouds seem to be related. These regions are outlined by the squares in Figures 12,13 , and 14, which are meant to indicate which $\mathrm{H}$ II regions were lumped together. They are numbered $1^{\prime}$ to $40^{\prime}$ to avoid confusion with the numbers of the $\mathrm{H}$ I holes. Note that sometimes two or more $\mathrm{H} \alpha$ features belong to the same 


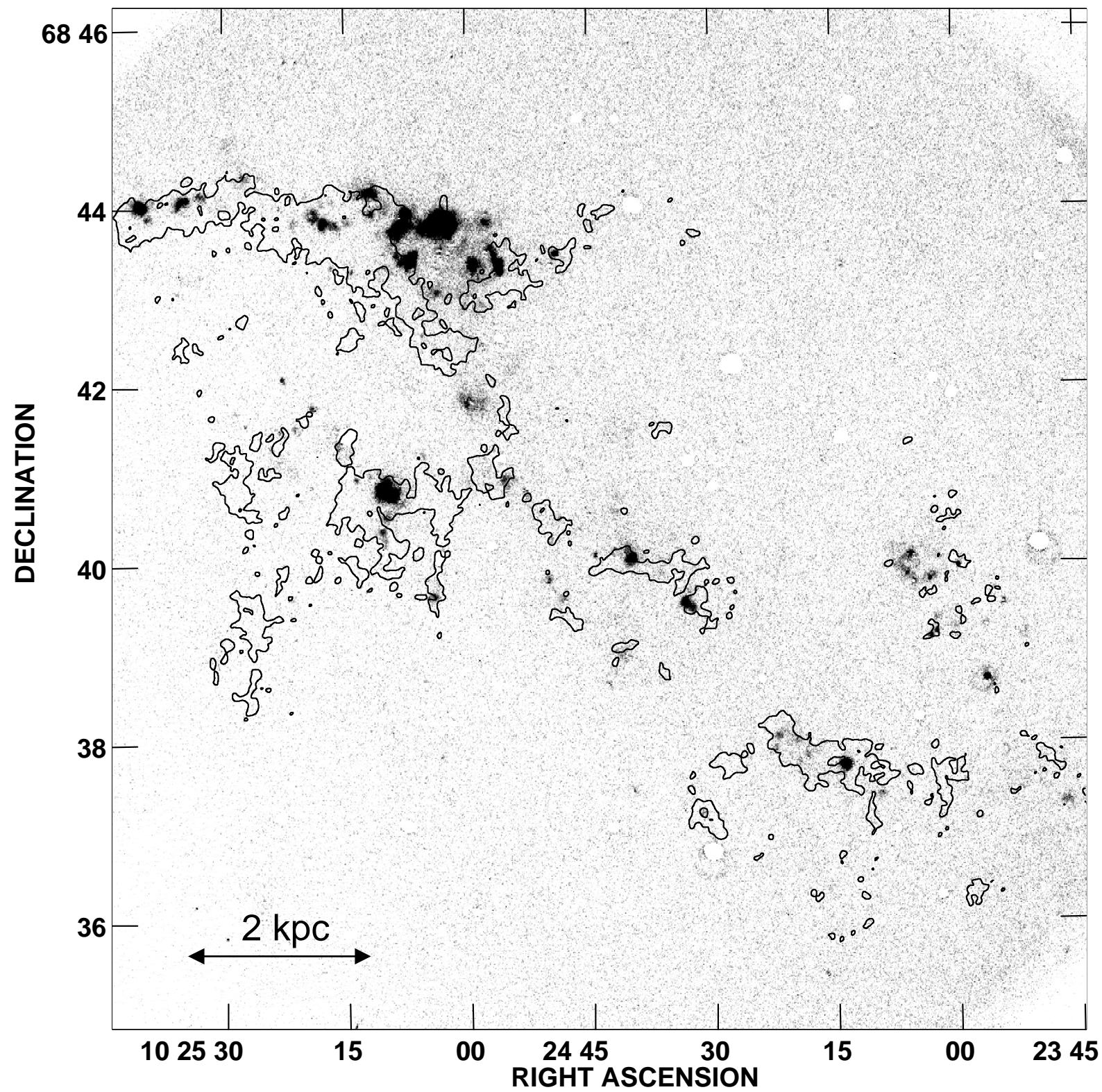

FIG. 11. $-\mathrm{H} \alpha$ image of IC 2574 overlaid with one contour of the $\mathrm{H}$ I surface-brightness map at $1.7 \times 10^{21}$ atoms $\mathrm{cm}^{-2}$. Note that regions where we find $\mathrm{H} \alpha$ emission coincide with high $\mathrm{H}$ I surface brightness.

$\mathrm{H}$ I structure (like a clump) and were thus considered as one region (e.g., in region $40^{\prime}$ ), whereas in other cases individual $\mathrm{H}$ II regions were treated as separate entities (e.g., regions $11^{\prime}$ and $\left.12^{\prime}\right)$. The $\mathrm{H} \alpha$ fluxes of the regions tell us something about the current SF rate and the average number density of the ionized gas, whereas the $\mathrm{H}$ I data yield approximations for the number density of neutral hydrogen, the velocity dispersion of the gas in that region, and the thresholds for SF. Figures 12, 13, and 14 are enlargements of the regions A-C marked in Figure 10 and display the regions defined by us. The position and size of the $\mathrm{H}$ I holes are plotted as well for comparison (please refer to Fig. 10 for cross-referencing of the holes). The fluxes of the $\mathrm{H}$ in regions were taken from the observations of MH94. Table 5 summarizes the cross-identifications between their and our regions. In this table we list the coordinates of the centers of our regions as well.

Table 6 summarizes the results of our analysis of the $\mathrm{H}$ II regions. The first column refers to the number of the $\mathrm{H}$ II region as defined in Figures 12,13, and 14. From the fluxes published by MH94, we deduced the total flux $F(\mathrm{H} \alpha)$ of our groups by simply summing their fluxes in the respective regions (col. [2], Table 6). From this number, the $\mathrm{H} \alpha$ luminosity can be calculated using $L(\mathrm{H} \alpha)=4 \pi D^{2} F(\mathrm{H} \alpha)$, which, in the case of IC $2574(D=3.2 \mathrm{Mpc})$, translates to $L(\mathrm{H} \alpha)=3.1 \times 10^{17} F(\mathrm{H} \alpha)$, where $F(\mathrm{H} \alpha)$ is given in ergs $\mathrm{cm}^{-2} \mathrm{~s}^{-1}$ and $L(\mathrm{H} \alpha)$ in units of solar luminosities $L_{\odot}$ $\left(1 L_{\odot}=3.85 \times 10^{33} \mathrm{ergs} \mathrm{s}^{-1}\right)$. The number of Lyman continuum photons per second is given by $N_{\text {Lyc }}=2.82$ $\times 10^{45} L(\mathrm{H} \alpha)$. Table 7 (see Devereux et al. 1997 and references therein) summarizes the expected properties of highmass stars; column (1) gives the spectral type, column (2) the stellar mass, column (3) the bolometric stellar luminosity, column (4) the number of Lyman continuum photons per second, column (5) the $\mathrm{H} \alpha$ luminosity $L(\mathrm{H} \alpha)$, and column (6) the ratio of bolometric to $\mathrm{H} \alpha$ luminosity. These equations and values can be used to estimate, for example, the equivalent number of $\mathrm{O} 5$ stars for each region (see below). 


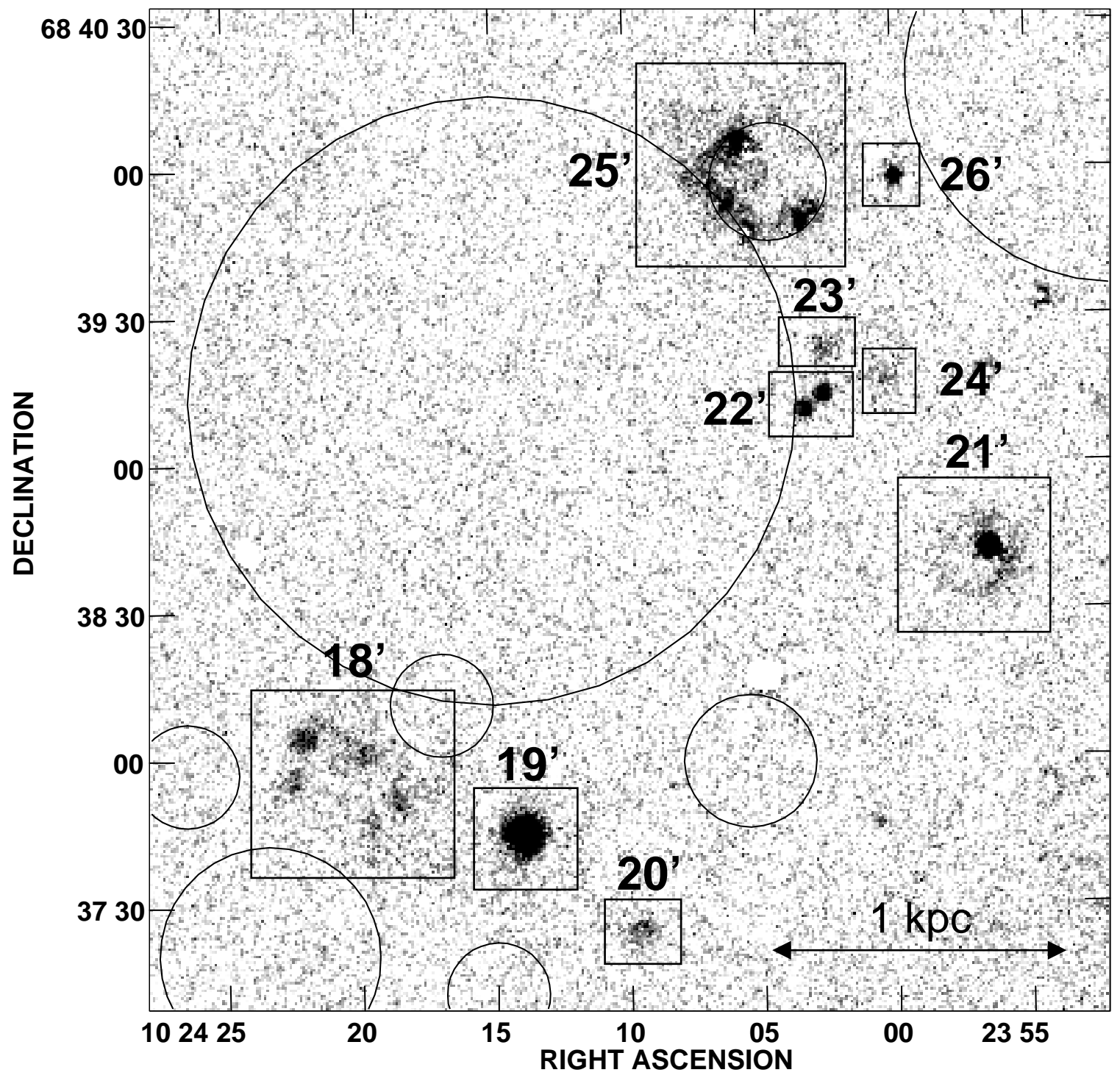

Fig. 12.-Enlargement of region A from Fig. 10. The circles indicate where we detected the $\mathrm{H}$ I holes (for identification of the $\mathrm{H} \mathrm{I}$ holes, please refer to Fig. 10). The boxes show how we defined groups of $\mathrm{H}$ II regions within IC 2574 (with sequence numbers).

Column (3) in Table 6 presents the volume density of the ionized free electrons over the area where we find $\mathrm{H} \alpha$ emission (as defined by MH94). This was arrived at by first calculating the emission measure (EM) of each of their regions according to:

$$
\mathrm{EM}=2.41 \times 10^{3} T^{0.92} \mathrm{~F}(\mathrm{H} \alpha) \mathrm{cm}^{-6} \mathrm{pc}
$$

(Peimbert, Rayo, \& Torres-Peimbert 1975), where $F(\mathrm{H} \alpha)$ is the $\mathrm{H} \alpha$ flux in units of ergs cm $\mathrm{cm}^{-2} \mathrm{~s}^{-1} \mathrm{sr}^{-1}$ and $T$ is assumed to be the canonical value of $10^{4} \mathrm{~K}$. From that, the mean electron density for every single region published by MH94 was derived using

$$
\mathrm{EM}=n_{e}^{2} d \mathrm{~cm}^{-6} \mathrm{pc}
$$

where $n_{e}$ is the electron density and $d$ the path length in pc through the $\mathrm{H}$ II region (assuming a spherical distribution of the $\mathrm{H} \alpha$ emission). Since the derived values for $n_{e}$ were very similar for a single region, we calculated the value for the electron density for each of our regions by taking an intensity-weighted average of the contributing MH94 regions. The average electron densities range from 0.5 to 2 $\mathrm{cm}^{-3}$. This is, most likely, a lower limit for the actual electron density due to internal absorption within IC 2574. Note that in the above, effects due to the filling factor of the gas are ignored. The real densities in the $\mathrm{H}$ II regions are likely much higher.

To derive properties of the $\mathrm{H}$ I features that are physically related to the $\mathrm{H}$ in regions, we defined areas $A$ around each of the $\mathrm{H}$ II regions with an effective radius given by $r_{\text {eff }}=$ $(A / \pi)^{-1 / 2}$, where $r_{\text {eff }}$ is given in parsecs (Table 6, col. [4]). Although this is a somewhat subjective process, we found that slight variations in the size of the aperture did not change the derived properties by more than $20 \%-30 \%$. Column (5) represents the total $\mathrm{H}$ I mass found within the aperture using the flux and primary beam corrected $\mathrm{H} \mathrm{I}$ data. For every single region, we measured the velocity dispersion of the $\mathrm{H}$ I by taking spectra through the $\mathrm{H}$ I cube. Column (6) summarizes the measured velocity widths defined as the FWHM from which the real velocity dispersion $\sigma_{v}$ (assuming a Gaussian velocity distribution) can be calculated using $\Delta v=2(2 \ln 2)^{-1 / 2} \quad \sigma_{v}=2.355 \quad \sigma_{v}$. Inter- 


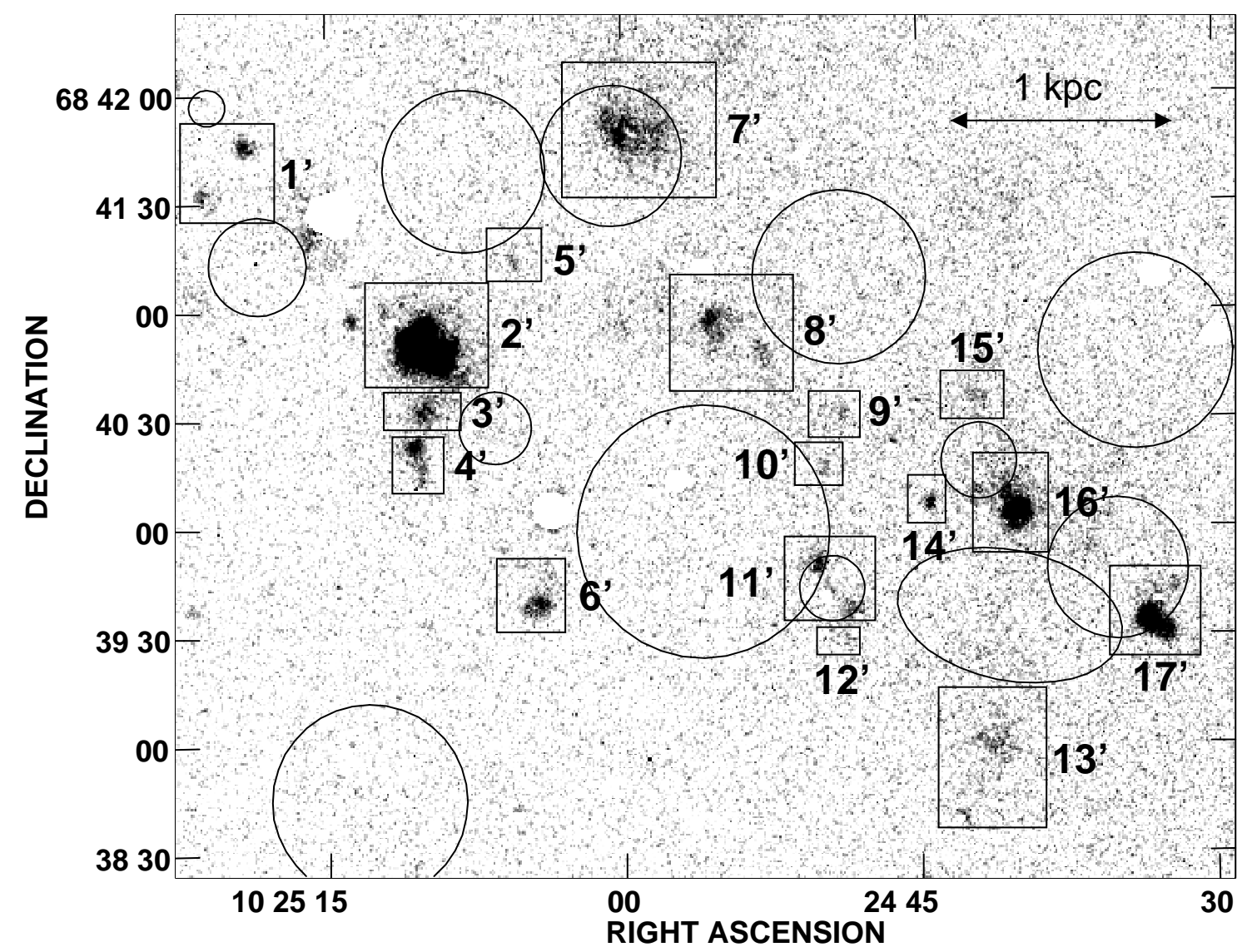

Fig. 13. - Enlargement of region B from Fig. 10. The circles indicate where we detected the $\mathrm{H}$ I holes (for identification of the $\mathrm{H}$ I holes, please refer to Fig. 10). The boxes show how we defined groups of $\mathrm{H}$ II regions within IC 2574 (with sequence numbers).

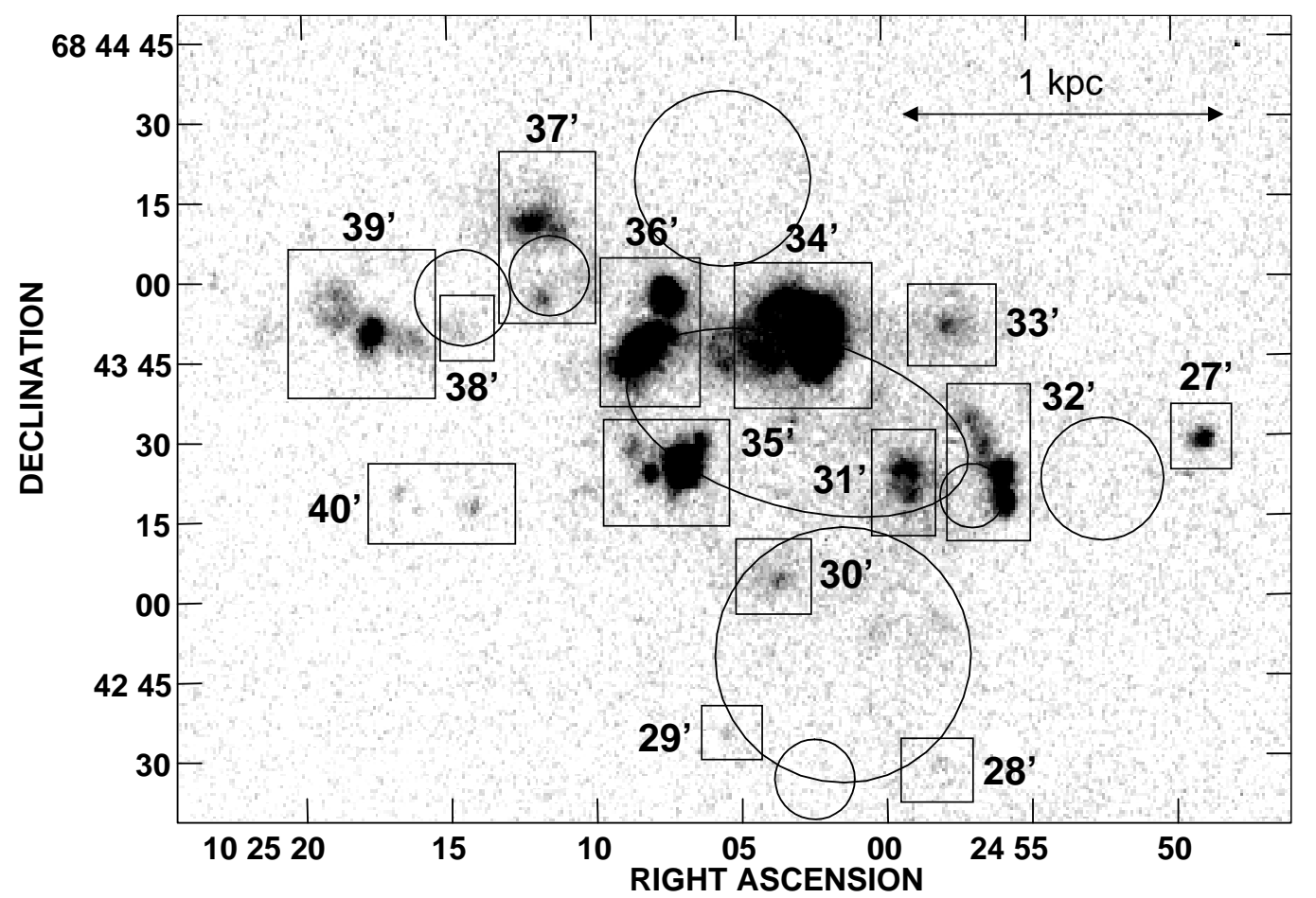

FIG. 14.-Enlargement of region $\mathrm{C}$ from Fig. 10. The circles indicate where we detected the $\mathrm{H}$ I holes (for identification of the H I holes, please refer to Fig. 10). The boxes show how we defined groups of $\mathrm{H}$ II regions within IC 2574 (with sequence numbers). 
TABLE 5

Cross-IDENTIFICATION OF OUR GRouping OF H in Regions With MH94

\begin{tabular}{|c|c|c|c|}
\hline Number & $\begin{array}{c}\alpha \\
(1950.0)\end{array}$ & $\begin{array}{c}\delta \\
(1950.0)\end{array}$ & Cross Identifications \\
\hline $1^{\prime}$. & 102520.2 & 684140 & 246,251 \\
\hline $2^{\prime} \ldots \ldots$. & 102510.1 & 684050 & $181,190,193,197,203,206,207,218,221,226,227,230$ \\
\hline $3^{\prime} \ldots \ldots$. & 102510.2 & 684032 & 208 \\
\hline $4^{\prime} \ldots \ldots$ & 102510.4 & 684019 & $195,213,214,227$ \\
\hline $5^{\prime} \ldots \ldots$ & 102505.7 & 684115 & 184 \\
\hline $6^{\prime} \ldots \ldots$ & 102504.5 & 683939 & 182,174 \\
\hline $7^{\prime} \ldots \ldots$ & 102459.5 & 684151 & $136,143,155,158,165$ \\
\hline $8^{\prime} \ldots \ldots$ & 102454.1 & 684053 & $125,126,131$ \\
\hline $9^{\prime} \ldots \ldots$ & 102448.9 & 684031 & 117 \\
\hline $10^{\prime} \ldots \ldots$ & 102449.9 & 684018 & 120 \\
\hline $11^{\prime} \ldots \ldots$ & 102449.5 & 683943 & 115,121 \\
\hline $12^{\prime} \ldots \ldots$ & 102448.7 & 683927 & 113,116 \\
\hline $13^{\prime} \ldots \ldots$ & 102441.3 & 683858 & $99,100,106$ \\
\hline $14^{\prime} \ldots \ldots$ & 102444.5 & 684007 & 111 \\
\hline $15^{\prime} \ldots \ldots$ & 102442.0 & 684037 & 105 \\
\hline $16^{\prime} \ldots \ldots$ & 102440.1 & 684005 & $94,96,102,104$ \\
\hline $17^{\prime} \ldots \ldots$ & 102433.1 & 683933 & $76,80,83,84$ \\
\hline $18^{\prime} \ldots \ldots$ & 102420.4 & 683755 & $57,58,60-63,65,67$ \\
\hline $19^{\prime} \ldots \ldots$ & 102413.9 & 683744 & 50 \\
\hline $20^{\prime} \ldots \ldots$ & 102409.3 & 683724 & 46 \\
\hline $21^{\prime} \ldots \ldots$ & 102357.0 & 683837 & $17,19,20$ \\
\hline $22^{\prime} \ldots \ldots$ & 102402.7 & 683912 & 30,33 \\
\hline $23^{\prime} \ldots \ldots$ & 102402.5 & 683923 & 31 \\
\hline $24^{\prime} \ldots \ldots$ & 102400.1 & 683917 & 25 \\
\hline $25^{\prime} \ldots \ldots$ & 102404.5 & 683958 & $24,28,32,34,36,38-41,43-46$ \\
\hline $26^{\prime} \ldots \ldots$ & 102359.7 & 683958 & 24 \\
\hline $27^{\prime} \ldots \ldots$ & 102449.1 & 684330 & 118 \\
\hline $28^{\prime} \ldots \ldots$ & 102458.4 & 684229 & 144 \\
\hline $29^{\prime} \ldots \ldots$ & 102505.7 & 684235 & 186 \\
\hline $30^{\prime} \ldots \ldots$ & 102503.9 & 684304 & 178 \\
\hline $31^{\prime} \ldots \ldots$ & 102459.4 & 684323 & 148 \\
\hline $32^{\prime} \ldots \ldots$ & 102456.1 & 684324 & $133,134,137,138$ \\
\hline $33^{\prime} \ldots \ldots$ & 102458.0 & 684352 & 140 \\
\hline $34^{\prime} \ldots \ldots$ & 102503.0 & 684351 & $167,169,179,180$ \\
\hline $35^{\prime} \ldots \ldots$ & 102507.2 & 684326 & 192,196 \\
\hline $36^{\prime} \ldots \ldots$ & 102508.4 & 684349 & 194, 198, 202 \\
\hline $37^{\prime} \ldots \ldots$ & 102512.0 & 684406 & $210,215,224,225$ \\
\hline $38^{\prime} \ldots \ldots$ & 102514.8 & 684351 & 235 \\
\hline $39^{\prime} \ldots \ldots$ & 102517.9 & 684351 & $237,238,240,242,244,245$ \\
\hline $40^{\prime} \ldots \ldots$ & 102515.7 & 684320 & $233,237,240$ \\
\hline
\end{tabular}

\footnotetext{
Note.-Units of right ascension are hours, minutes, and seconds, and units of declination are
} degrees, arcminutes, and arcseconds.

estingly, these values tend to be high where two or more shells overlap (e.g., the H II complexes 16, 31, 32, 33). These high dispersions are probably caused by blending along the line of sight of these shells.

Column (7) presents the average density of the neutral hydrogen, which was calculated using

$$
\rho_{\mathrm{HI}}=M_{\mathrm{HI}} /\left(\frac{4}{3} \pi r_{\mathrm{eff}}^{3}\right),
$$

with $M_{\mathrm{HI}}$ and $r_{\text {eff }}$ the values from columns (5) and (4), respectively. Values for $\rho_{\mathrm{HI}}$ range from about $1 \mathrm{~cm}^{-3}$ to values as high as $6 \mathrm{~cm}^{-3}$. Note that these are only averages and that locally the $\mathrm{H}$ I density might be more enhanced. Based on the analysis by MCR94 and some crude mass modeling $(\S 3.3)$ we derive an average density in the disk of $0.008 M_{\odot} \mathrm{pc}^{-3}=0.3$ atom $\mathrm{cm}^{-3}$. The regions in which star formation is currently active thus have an overdensity of an order of magnitude (see Table 6).

The average surface density of the $\mathrm{H}$ I regions is shown in column (8). Note that virtually all values are larger than
$1 \times 10^{21} \mathrm{~cm}^{-2}$, which is in excellent agreement with the observational value given by Skillman (1987). In other words, $\mathrm{SF}$ is taking place if the projected $\mathrm{H}$ i surface density reaches values of about $10^{21} \mathrm{~cm}^{-2}$ not only for a resolution of $500 \mathrm{pc}$, as observed by Skillman, but also for almost an order of magnitude improvement $(\approx 95 \mathrm{pc})$ ! The highest surface density found is of order $2.5 \times 10^{21} \mathrm{~cm}^{-2}$.

Finally, column (9) summarizes some remarks regarding the regions. Most of the $\mathrm{H}$ in regions are related to the rim of an $\mathrm{H}$ I shell. It should be noted at this point that in the course of checking for correlations between $\mathrm{H} \alpha$ and $\mathrm{H}$ I emission, we detected five new tiny holes at the positions were there is $\mathrm{H} \alpha$ emission. However, because these holes were not detected in our inspection of the $\mathrm{H}$ I cubes, we did not include them in the final catalog, which is thought to be complete only for holes with radii larger then about $100 \mathrm{pc}$ (as discussed in $\S \S 4.1$ and 6.2).

We cataloged four of the $\mathrm{H}$ I features as "clumps" (regions $1^{\prime}, 2^{\prime}, 6^{\prime}$, and $22^{\prime}$ ). By "clump" we mean a well- 
TABLE 6

Derived Properties of Grouped H il Regions

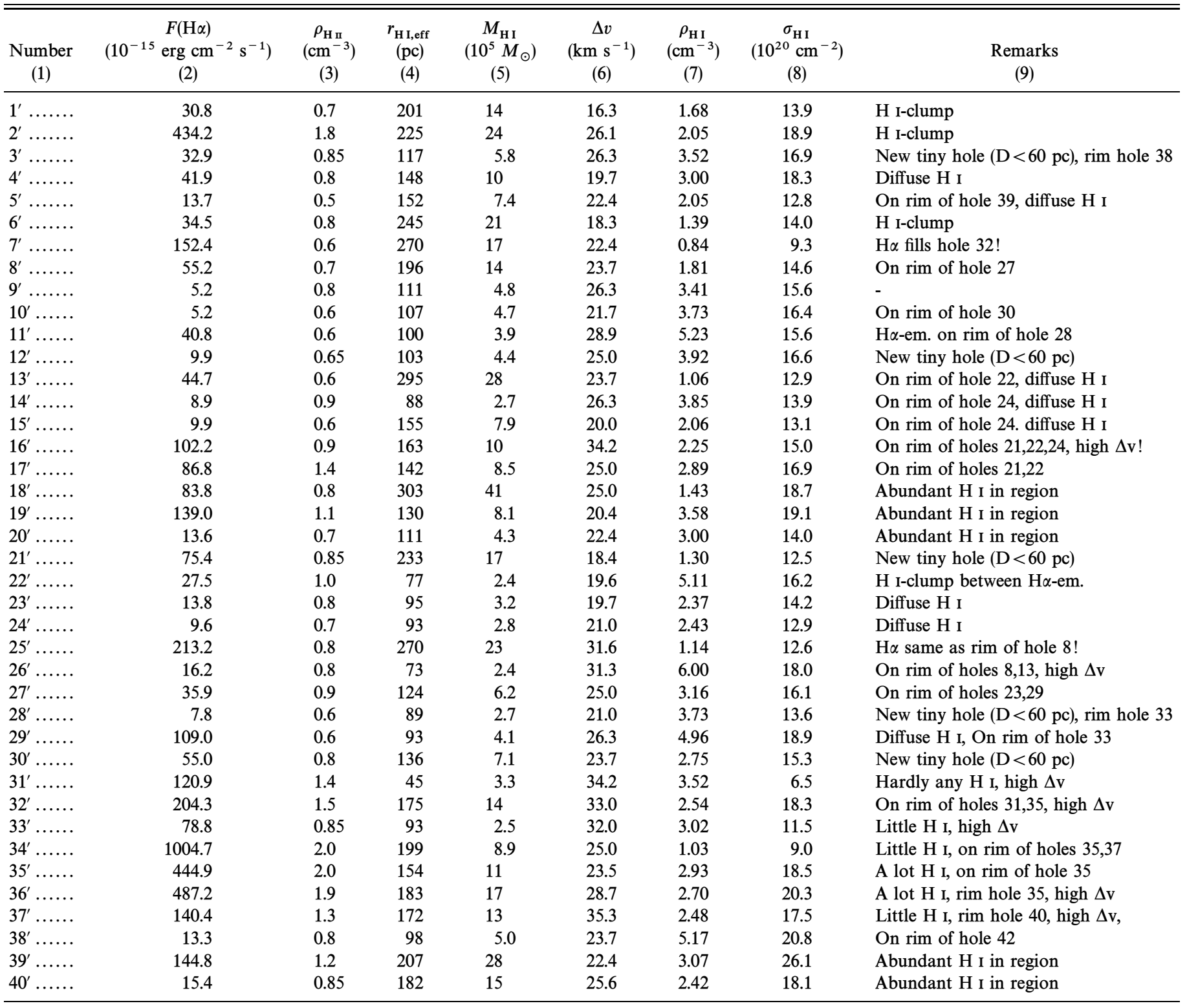

defined $\mathrm{H}$ I region in two spatial dimensions as well as in velocity space. These clumps look like small, filled circles in position-velocity space. For those cases we asked ourselves whether these clumps are gravitationally confined or not. To answer that question, we calculated the virial masses of these objects using

$$
M_{\text {vir }}=250 \Delta v^{2} r_{\text {eff }} M_{\odot}
$$

(Rohlfs \& Wilson 1996), where $\Delta v$ is the FWHM in $\mathrm{km} \mathrm{s}^{-1}$ and $r_{\text {eff }}$ the radius of the clump in parsecs. The virial masses

TABLE 7

Some Properties of High-Mass Stars ${ }^{\mathrm{a}}$

\begin{tabular}{cccccc}
\hline \hline $\begin{array}{c}\text { Type } \\
(1)\end{array}$ & $\begin{array}{c}\text { Mass }\left(M_{\odot}\right) \\
(2)\end{array}$ & $\begin{array}{c}L_{\text {bol }}\left(L_{\odot}\right) \\
(3)\end{array}$ & $N_{\text {Lyc }}$ & $\begin{array}{c}L(\mathrm{H} \alpha) \\
(4)\end{array}$ & $\begin{array}{c}L_{\text {bol }} / L(\mathrm{H} \alpha) \\
(5)\end{array}$ \\
\hline O5 $\ldots \ldots$ & 40 & $6.8 \times 10^{5}$ & $4 \times 10^{49}$ & $1.4 \times 10^{4}$ & 49 \\
O7 $\ldots \ldots$. & 25 & $1.0 \times 10^{5}$ & $4 \times 10^{48}$ & $1.4 \times 10^{3}$ & 71 \\
O9 $\ldots \ldots$. & 20 & $4.6 \times 10^{4}$ & $1.3 \times 10^{48}$ & $4.6 \times 10^{2}$ & 100 \\
B0 $\ldots \ldots$. & 16 & $2.5 \times 10^{4}$ & $2.5 \times 10^{47}$ & 89 & 282 \\
B1 $\ldots \ldots$. & 10 & $5.2 \times 10^{3}$ & $1.9 \times 10^{45}$ & 0.67 & $7.8 \times 10^{3}$ \\
B2 $\ldots \ldots$. & 8 & $2.9 \times 10^{3}$ & $4.5 \times 10^{44}$ & 0.16 & $1.8 \times 10^{4}$ \\
B5 $\ldots \ldots$. & 6 & $7.9 \times 10^{2}$ & $4.8 \times 10^{43}$ & 0.02 & $4.0 \times 10^{4}$ \\
\hline
\end{tabular}

a Based on Devereux et al. 1997. 
of the four regions are: $M_{\text {vir }}\left(1^{\prime}\right)=13 \times 10^{6} M_{\odot}, M_{\text {vir }}\left(2^{\prime}\right)=$ $38 \times 10^{6} M_{\odot}, M_{\text {vir }}\left(6^{\prime}\right)=21 \times 10^{6} M_{\odot}$, and $M_{\text {vir }}\left(22^{\prime}\right)=7$ $\times 10^{6} M_{\odot}$. As we see, the virial masses are all about one order of magnitude higher than the $\mathrm{H}$ I masses. So, the clumps are either not gravitationally bound or they are held together by as yet undetected material. One possibility is that they contain an order of magnitude more mass in the form of molecules. This will be hard to confirm, however, as dwarf galaxies have been notoriously difficult to detect in $\mathrm{CO}$, the most abundant tracer for molecular gas (e.g., Sage et al. 1992). Dark matter (DM) could play a role, but the mass density, including DM, near the above mentioned SF regions is of order $0.008 M_{\odot} \mathrm{pc}^{-3}$ (see $\S 3.3$ ), insufficient by a wide margin.

If the observed velocity dispersion is not due to gravitation, the question remains, why do we see clumps? We can think of two more mechanisms, magnetic confinement or pressure. The former is unlikely to play a large role. Dwarf galaxies do not possess large-scale magnetic fields (the exceptions being NGC 4449 and IC 10; e.g., Chyzy et al. 1998 ), any nonthermal radio continuum radiation being confined to the strongest regions of active star formation (Deeg et al. 1993).

We did not find any correlation of $\mathrm{H} \alpha$ flux with the surrounding $\mathrm{H}$ I density. However, one would not really expect any strong correlation of this kind. Not only is the $\mathrm{H} \alpha$ emissivity of a star-forming region strongly dependent on the age of the region (see, e.g., Gerritsen \& Icke 1997), but it is also dangerous to try to deduce actual physical properties of the SF regions from mean values, such as average electron or $\mathrm{H}$ I densities, while ignoring spatial variations in their volume filling factors.

In some regions within IC 2574 (e.g., region 40') we find high $\mathrm{H}$ I surface densities but only little $\mathrm{H} \alpha$ emission, indicating that possibly a major star formation burst is about to commence there and that therefore most of the gas is still neutral. With the help of the equations given above, we can calculate approximately how many stars are needed to create the observed $\mathrm{H} \alpha$ flux. In the case of region $40^{\prime}$, we find a flux of $1.5 \times 10^{-14} \mathrm{ergs} \mathrm{cm}^{-2} \mathrm{~s}^{-1}$ and deduce a luminosity of $4.7 \times 10^{3} L_{\odot}$, which is the equivalent of a few O7 stars only (see Table 7). Note, however, that there also exist regions where the situation is reversed and most of the $\mathrm{H} \mathrm{I}$ is ionized by the Lyman continuum photons of the most massive stars of the powerful $\mathrm{H}$ II regions. Region $34^{\prime}$ is a prominent example (see also Fig. 11). From the $\mathrm{H} \alpha$ flux of $F(\mathrm{H} \alpha) \approx 1 \times 10^{-12} \mathrm{ergs} \mathrm{cm}^{-2} \mathrm{~s}^{-1}$, we derive an $\mathrm{H} \alpha$ luminosity of $L(\mathrm{H} \alpha)=3.1 \times 10^{5} L_{\odot}$, which corresponds to an equivalent of $22 \mathrm{O} 5$ stars - in other words, a major SF region.

\section{DISCUSSION}

\subsection{What Created the $\mathrm{H}$ i Holes?}

The ISM of IC 2574 is dominated by expanding H I holes and shells. Similar structures have been found in other gasrich dwarf galaxies (e.g., Holmberg II (PWBR92) and IC 10 (Wilcots \& Miller 1998), and in more massive spiral galaxies like our Galaxy (Heiles 1979), M31 (Brinks \& Bajaja 1988), and M33 (Deul \& den Hartog 1990). One of the major questions concerning these structures is what physical process or processes are responsible for their origin.

Heiles (1979), who was the first to recognize expanding $\mathrm{H}$ I shells in the Galaxy, concluded that "many of the H I structures $[\ldots]$ were probably produced by large amounts of energy injected directly to the interstellar medium." One obvious mechanism is the aftereffects of massive star formation: strong stellar winds and supernova explosions (see the review by Tenorio-Tagle \& Bodenheimer 1988). Detailed numerical modeling by various authors (e.g., Palouš, Franco, \& Tenorio-Tagle 1990; Silich et al. 1996; Palouš, Tenorio-Tagle, \& Franco 1994) has strengthened confidence in this explanation, as has the work by Oey \& Clarke (1997) who in a (semi-) analytical way managed to predict the H I shell size distribution in the SMC based on the observed $\mathrm{H} \alpha$ luminosity function.

Having said that, there are potentially problems with this interpretation, as mentioned by Radice, Salzer, \& Westpfahl (1995) and Rhode, Salzer, \& Westpfahl (1999). Looking for remnant stellar clusters at the centers of several large holes in Holmberg II they failed to find a population of A and F main-sequence stars. Moreover, it is difficult to imagine that the largest $\mathrm{H}$ I shells which are located beyond the optical galaxy should have been created by star formation (an argument that holds equally well for some of the holes in IC 2574). This view is corroborated by ultraviolet imaging of Holmberg II by Stewart et al. (1999), who finds no FUV emission from the outer regions of Holmberg II.

It should be noted, however, that the nondetection of population $\mathrm{A}$ and $\mathrm{F}$ stars does not necessarily mean that none of the $\mathrm{H}$ I are created by SF. In dwarf galaxies, in the absence of shear, $\mathrm{H}$ I shells can reach large dimensions and grow so old that no obvious bright cluster remnant will remain visible in the center (Efremov et al. 1998). Some of the observed supershells are so old that the brightest members of stellar cluster that created the hole may have evolved off the main sequence and dispersed (assuming a similar IMF as in the Galaxy). This may make a detection of old clusters difficult. Such a case (LMC 4/Constellation III) is described by Efremov \& Elmegreen (1998).

Another potential problem with the conventional picture of the origin of the holes is that for the largest ones the energy requirements seem to surpass the amount that can possibly be released into the ambient ISM via stellar winds and supernova explosions. The giant shell in M101 (van der Hulst \& Sancisi 1988) is just one example of such a structure. Tenorio-Tagle et al. (1987) proposed as an alternative the infall of high-velocity clouds, which could deposit an amount of energy of $10^{52}$ to $10^{54}$ ergs per collision (see the review by Tenorio-Tagle \& Bodenheimer 1988 for further references). Even though this mechanism seems a viable explanation for at least some holes, it is unlikely that it plays a dominant role. Certainly it cannot be as important in dwarf galaxies because of their much smaller collisional cross section.

Recently, an alternative explanation for the creation of supergiant $\mathrm{H}$ I shells in galaxies has been proposed, namely, Gamma Ray Bursters (GRBs; Efremov et al. 1998; Loeb \& Perna 1998). Even though the basic physics of GRBs is not yet known the picture to emerge is that they are highly energetic events (of order $10^{54}$ ergs) which release huge amounts of energy in a very short period of time (order of a few seconds).

Although we recognize that perhaps not all holes can be explained by stellar winds and supernova explosions, we feel that there is sufficient evidence for the conventional interpretation to warrant an analysis in those terms, which is what we undertake in the rest of this paper. 


\subsection{Statistical Properties of the Holes: Comparison with Other Galaxies}

For the first time we are now in a position to address an important question regarding the creation and evolution of holelike structures in the ISM: how are their properties related to their environment, i.e., the type of host galaxy. In what follows we will compare the observed and derived $\mathrm{H} \mathrm{I}$ hole properties of IC 2574 with those found in M31 (Brinks \& Bajaja 1986), an example of a massive spiral galaxy similar to our own, M33 (Deul \& den Hartog 1990), a less massive spiral, and Ho II (PWBR92), another dwarf galaxy in the same group of galaxies but 4 times less massive than IC 2574. In other words, the sequence M31-M33-IC 2574-Ho II spans a large range of different Hubble types, from massive spirals to low-mass dwarfs.

Despite the fact that a similar study, although at considerably higher sensitivity, is now available for the SMC (Staveley-Smith et al. 1997), we decided not to include their results. The main reason is that the linear scales (or spatial frequencies) sampled by the ATNF hardly overlap with those observed in the galaxies listed above. The linear resolution, at $28 \mathrm{pc}$, is almost four times higher than, for example, the VLA maps on IC 2574. At the other end of the spectrum, because of the lack of short spacing information, structures larger than a few hundred parsecs will have been missed. Moreover, the SMC is a very disturbed system, being torn apart by tidal forces caused by interactions with the LMC and the Galaxy.

Limiting the comparison to the four galaxies listed above has some further advantages. The linear resolutions are very similar, as are the velocity resolutions with which they have been observed (see Table 8 for a summary). In addition, all four galaxies were examined in more or less the same fashion, one of the authors (E. B.) having taken part in the analysis of three of the four objects. We are aware that the results for the four galaxies suffer partially from low statistics and incompleteness due to personal bias and observational constraints (such as the beam size). However, we feel that these effects, to first order, affect a comparison in a similar way and that it is valid to try to find global trends as a function of Hubble type.

For the sake of brevity, rather than first show the statistical properties of the holes in IC 2574 separately, we immediately go to a comparison with the other three objects. In Figures 15, 16, 17, 18, and 19 we compare the distributions of swept-up matter, the energies, diameters, expansion velocities, and ages of the holes in the four gal-

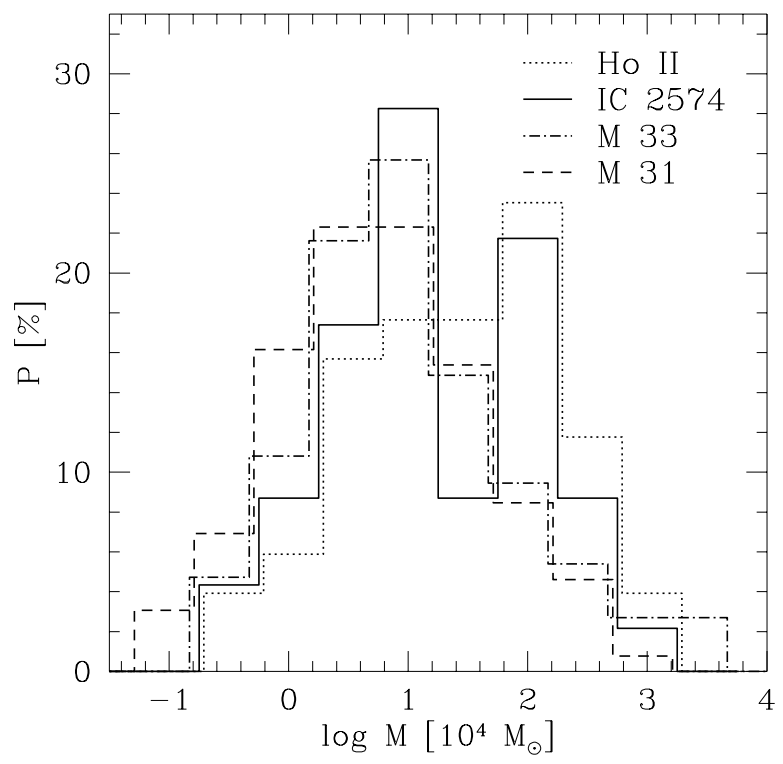

FIG. 15.-Comparison of the relative percentage distribution of the indicative masses of the H I holes in Ho II, IC 2574, M33, and M31.

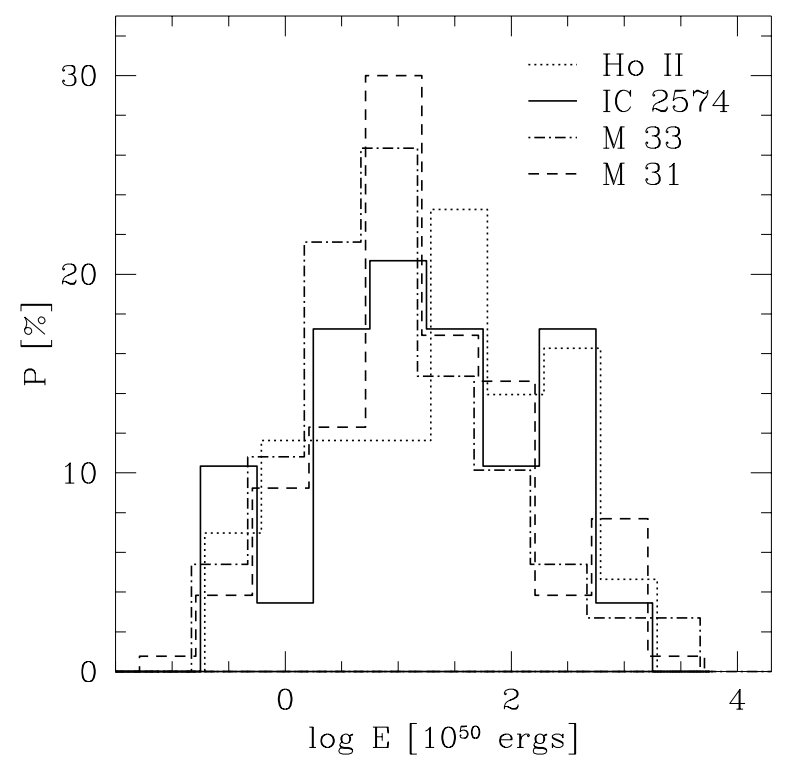

FIG. 16. - Comparison of the relative percentage distribution of the energies required to produce the $\mathrm{H}_{\mathrm{I}}$ holes in Ho II, IC 2574, M33, and M31.

TABLE 8

Summary of H i Hole Statistics in Four Nearby GalaXies

\begin{tabular}{ccccc}
\hline \hline Property & M31 & M33 & IC $2574^{\mathrm{c}}$ & Ho II $^{\mathrm{d}}$ \\
\hline Linear resolution $(\mathrm{pc}) \ldots \ldots \ldots \ldots \ldots \ldots \ldots$ & 100 & 55 & 95 & 65 \\
Velocity resolution $\left(\mathrm{km} \mathrm{s}^{-1}\right) \ldots \ldots \ldots \ldots \ldots$ & 8.2 & 8.2 & 2.6 & 2.6 \\
Average surface density $\left(10^{20} \mathrm{~cm}^{-2}\right) \ldots \ldots$ & 5 & 9 & 4 & 10 \\
Average volume density $\left(\mathrm{cm}^{-3}\right) \ldots \ldots \ldots \ldots$ & 0.6 & 0.45 & 0.15 & 0.2 \\
Average velocity dispersion $\left(\mathrm{km} \mathrm{s}^{-1}\right) \ldots \ldots$ & 8 & 8 & 7 & 7 \\
Derived scaleheight $(\mathrm{pc}) \ldots \ldots \ldots \ldots \ldots \ldots \ldots$ & 120 & 100 & 350 & 625 \\
Number of holes $\ldots \ldots \ldots \ldots \ldots \ldots \ldots \ldots \ldots$ & 141 & 148 & 48 & 51 \\
Sensitivity per channel $\left(10^{20} \mathrm{~cm}^{-2}\right) \ldots \ldots \ldots$ & 0.3 & 1.0 & 0.5 & 2.4 \\
\hline
\end{tabular}

\footnotetext{
${ }^{a}$ Brinks \& Bajaja 1986.

b Deul \& den Hartog 1990.

c This paper.

d Puche et al. 1992.
} 


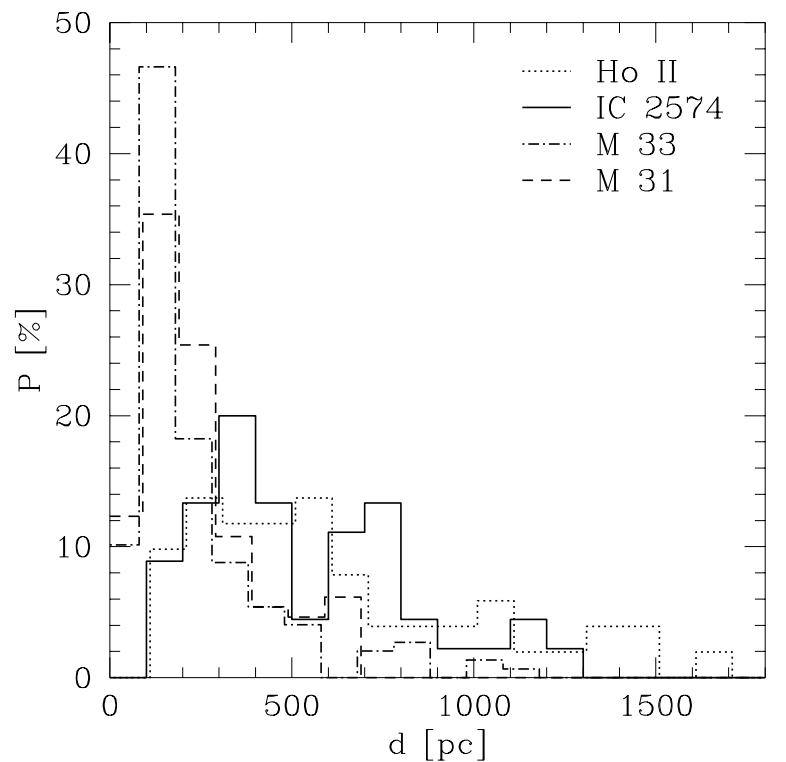

FIG. 17.-Comparison of the relative percentage distribution of the diameters of the H I holes in Ho II, IC 2574, M33, and M31.

axies. In each graph we plot in the form of histograms the relative number of holes, in percentages, in order to make a direct comparison possible. In each histogram, the data for the individual objects are binned in the same way. To improve the presentation, however, the respective bins were slightly shifted when plotting the results.

Figure 15 shows an overlay of the indicative masses of the $\mathrm{H}$ I holes, binned logarithmically. The values range from typically $10^{4}$ to $5 \times 10^{6} M_{\odot}$. Even though a two-sample Kolmogorov-Smirnov test rejects the hypothesis of pairwise equality of the distributions, a casual inspection shows that the indicative masses span roughly the same range. The mean masses (in $10^{5} M_{\odot}$ ) for the dwarf galaxies (Ho II: 10.5 ,

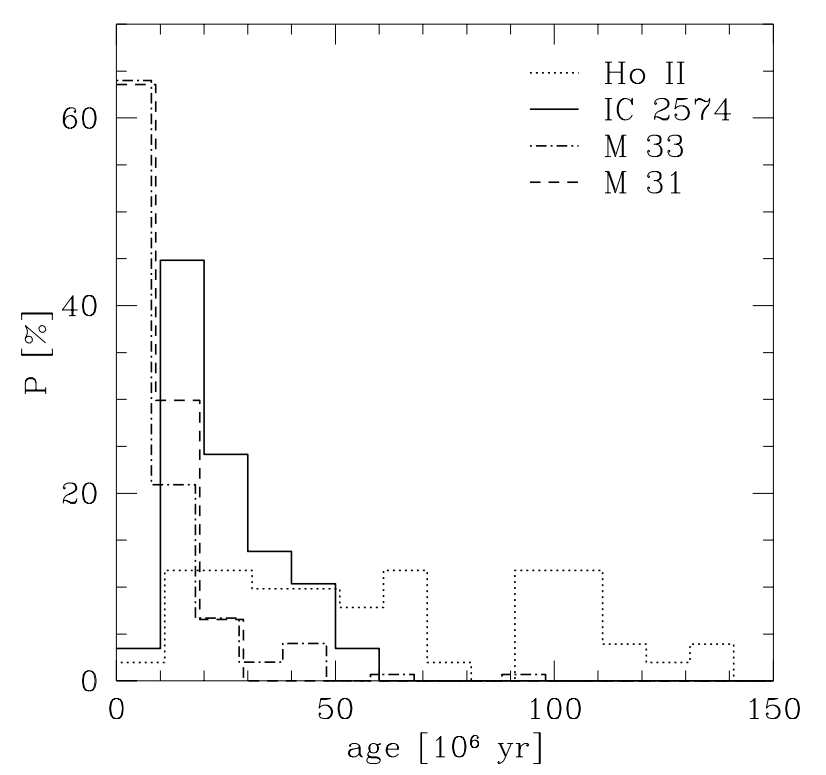

FIG. 18.-Comparison of the relative percentage distribution of the ages of the $\mathrm{H}$ I holes in Ho II, IC 2574, M33, and M31.

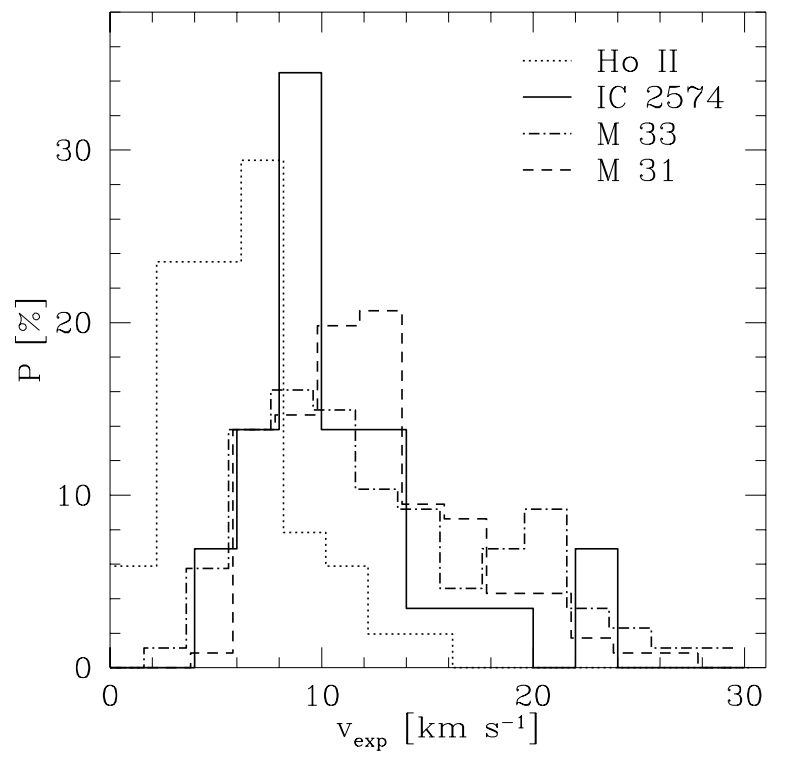

Fig. 19.-Comparison of the relative percentage distribution of the expansion velocities of the $\mathrm{H}$ I holes in Ho II, IC 2574, M33, and M31.

IC 2574: 8.4) are slightly higher as compared to the spirals (M33: 4.1, M31: 3.2). This is somewhat surprising if one realizes that the volume density in the plane of a spiral galaxy is typically higher by a factor of 3 as compared with that found in a dwarf galaxy. $\mathrm{H}$ I column densities have a much smaller variation from object to object, however (see Table 8), which probably explains why the swept-up matter works out to be more or less the same. As a warning, it should be borne in mind that, in order to determine the $\mathrm{H} \mathrm{I}$ densities, we smeared out any fine-scale structure, potentially adding somewhat to the errors.

Figure 16 is similar to Figure 15, showing instead the distribution of the logarithm of the energies needed to create the observed $\mathrm{H}$ I holes (under the assumption that they were created by supernova explosions). The derived energies range from $10^{50}$ to $10^{53}$ ergs. We find the important result that the distributions are identical, at least to first order (mean energies [10 ${ }^{52}$ ergs]: Ho II: 1.4, IC 2574: 1.1, M33: 1.6, M31: 1.5). A two-sample Kolmogorov-Smirnov test reveals that there is no significant pair-wise difference at the $95 \%$ level between the four energy distributions. From this we conclude that regardless of Hubble type of the parent galaxy, the star clusters (or whatever other process created the $\mathrm{H}$ i holes) deposit more or less the same amount of energy into the ISM.

Figure 17 shows an overlay of the size distribution of the holes found in the four galaxies. In this and the following plots the bins are on a linear scale. Note that there is a clear difference with Hubble type! The size distribution for holes in M31 and M33 cuts off sharply near 600 pc. In contrast, holes in IC 2574 and Ho II reach sizes of 1200 to 1500 pc, respectively. The lack of holes with sizes smaller than about $100 \mathrm{pc}$ is due to our resolution limit. The fact that the holes get larger for "later" Hubble types (i.e., galaxies with lower mass) can be understood as follows. Smaller galaxies have lower masses and their mass surface density in the disk is lower as well. The one-dimensional velocity dispersion varies little, if any, among galaxies. We find in the four objects under scrutiny, taking into account an uncertainty 
of $\pm 1 \mathrm{~km} \mathrm{~s}^{-1}$, a range of 6-9 $\mathrm{km} \mathrm{s}^{-1}$. This, combined with the lower mass surface density, leads to dwarf galaxies having much thicker disks with aspect ratios of 1:10 rather than 1:100. Hence, for the same amount of energy deposited, as argued above, an $\mathrm{H}$ I shell can grow much larger, because of a lower gravitational potential and a lower ambient density. Moreover, in dwarf galaxies shells are prevented from breaking out of the disk because it is thicker (see $\S 3.3$ ).

The age distribution of the holes (Fig. 18) also shows a sequence with Hubble type. The mean values for the ages of the holes are: $10 \times 10^{6}$ years (M31); $12 \times 10^{6}$ years (M33); $23 \times 10^{6}$ years (IC 2574), and $62 \times 10^{6}$ years (Ho II). In the spirals no holes are found that are older than $30 \mathrm{Myr}$ whereas the distribution in dwarfs is flatter and spans an age range of up to $120 \mathrm{Myr}$. This is not unexpected, because in more massive galaxies shear and mixing due to the passage of density waves tend to destroy holes. In dwarfs, unless a shell is hit by a neighboring explosion and subsequently expanding structure, a feature can potentially persist for a long time, until the rim disperses and mixes with the surrounding ISM.

Finally, the distribution of the expansion velocities is presented in Figure 19. The predominantly low values for the dwarfs (especially Ho II) reflect, again, the fact that we see many large holes in the dwarfs that have almost stalled. A word of caution should be added, because the velocity resolution of the IC 2574 and Ho II observations was a factor of about 3 better than those of M31. Very young (i.e., smaller than $\sim 100 \mathrm{pc}$ ) and fast expanding holes are missed, of course, but this holds true for all galaxies in the sample.

Figure 20 displays an alternative way of looking at the properties of the $\mathrm{H}$ I holes on a galaxy-wide scale. It shows the expansion velocities of the shells in the four nearby galaxies plotted as a function of their diameter (crosses). Based on the hydrodynamical models for expanding shells (Chevalier 1974), we plotted lines of constant energy for the expanding structures in Figure 20 (assuming a constant density of the ambient medium of $0.15 \mathrm{~cm}^{-2}$ ). The plotted curves represent energies at a level of $10^{49}, 10^{50}, 10^{51}$, and $10^{52}$ ergs, respectively ( from left to right). The evolution of an individual hole of a given energy is along an equi-energy line from the top of the plot downward. In addition, lines of equal ages are shown in the figure, ranging from $10^{7}$ years (steepest slope) to $5 \times 10^{7}$ years and $10^{8}$ years.

There is a marked difference between the two spiral galaxies and the dwarfs. The $\mathrm{H}$ I shells in M31 and M33 are smaller and the range of expansion velocities is larger. In fact, looking at the objects in order of decreasing mass (M31-M33-IC 2574-Ho II), there seems to be a gradually shifting pattern. Whereas in the larger spirals there is an absence of older holes (older than a few $\times 10^{7}$ years), holes in IC 2574 range between $5 \times 10^{7}$ and $10^{8}$ years, and holes in Ho II cluster range near $10^{8}$ years.

This suggests an alternative explanation for why we do not see younger holes in IC 2574 and Ho II. The SF his-

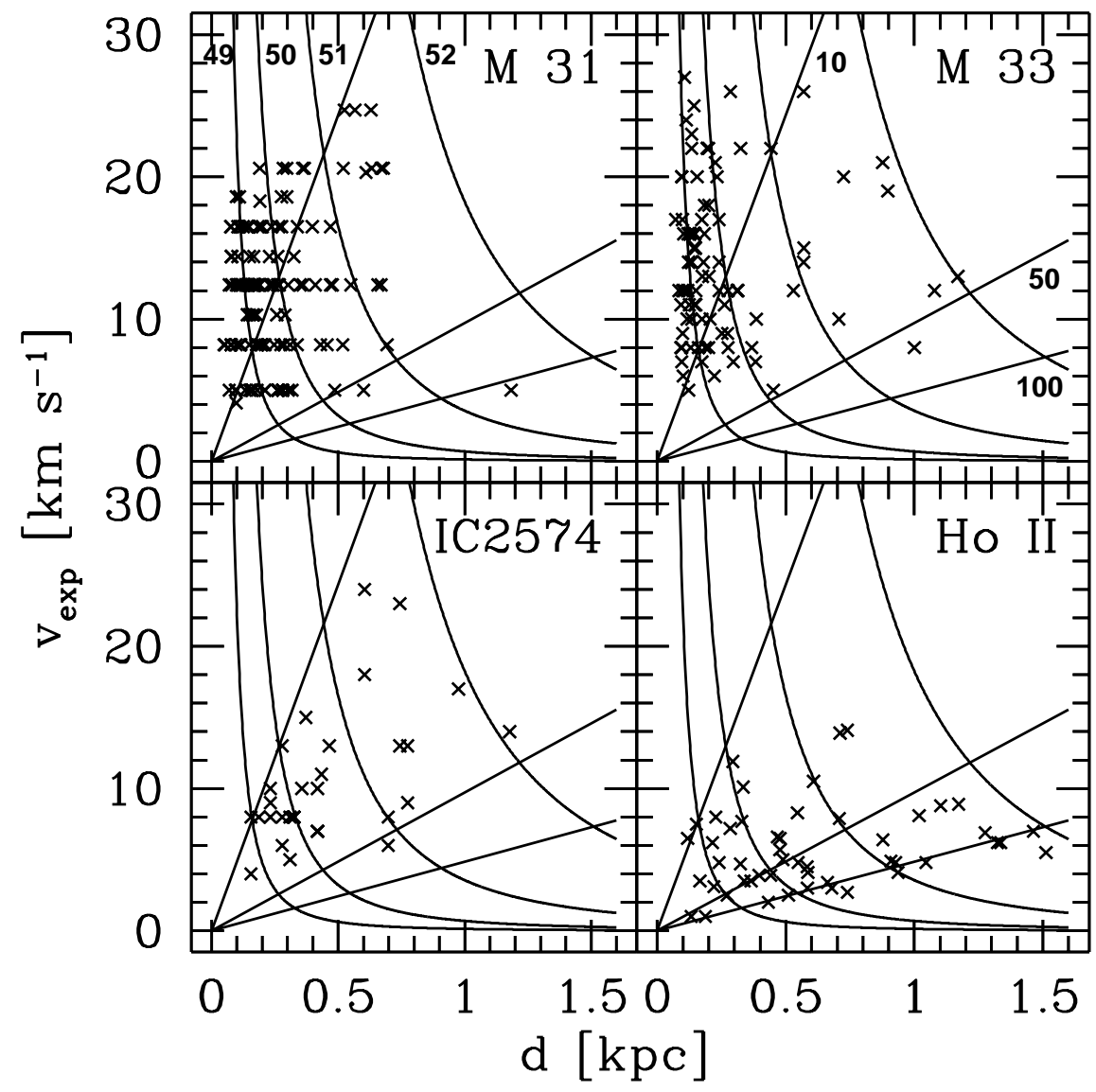

FIG. 20.- Relation between expansion velocity and diameter in each of the four galaxies. Crosses represent observed values. The plotted curves are lines of constant energy (based on Chevalier 1974) at levels of $10^{49}, 10^{50}, 10^{51}$, and $10^{52} \mathrm{ergs}$ ( from left to right). The straight lines correspond to constant age and are drawn at $10^{7}$ years (steepest slope), $5 \times 10^{7}$ years, and $10^{8}$ years. 
tories of these dwarf galaxies could be quite different from the spirals. In the latter, the global SF rate is, to first order, constant with time. Therefore, there is always a population of young holes. In contrast, in gas-rich dwarf irregulars, SF occurs in bursts that transform them into $\mathrm{H}$ II or blue compact dwarf (BCD) galaxies. It might be that IC 2574 and Ho II have undergone a recent burst, perhaps triggered by a close passage with another member of the M81 group, and are now in their post-starburst phase and that this is why we do not see many young holes. Our data alone do not allow us to choose between the two possibilities.

Oey \& Clarke (1997), using standard adiabatic shell evolution, predict the size distribution for the populations of OB superbubbles. In their paper they compare their predictions with observations of the SMC and the same three galaxies with which we compared our IC 2574 data, namely, M31, M33, and Ho II. Without going into detail, their prediction fits the observations for the SMC surprisingly well. This is true despite the fact that they ignore the selection effect (missing short spacings) which renders the SMC maps insensitive to the large structures that should dominate the appearance of dwarf galaxies. Also, they emphasize stalled shells, i.e., objects that have reached their largest diameter. This is in contradiction to the fact that the SMC shells, especially the smaller ones, are all found to be rapidly expanding. Their approach works less well for the other objects. They ascribe this to the data being incomplete, not only when considering the smallest detectable shells, but overall because of the reduced sensitivity as compared with the SMC data. In that respect, our IC 2574 are not better than the maps on M31, M33, or Ho II. Hence, a thorough test of Oey \& Clarke's predictions will have to await higher resolution and higher sensitivity data on the nearest (dwarf) galaxies.

\subsection{Intrinsic Shape of the Shells}

One of the results from our analysis is that over $90 \%$ of the holes are well approximated by a circular shape (the few exceptions are discussed in $\S 4.2$ ). This is a major difference with other galaxies studied thus far in similar detail (i.e., Ho II, M31, M33, M101, NGC 6946), which tend to have much more elliptical holes. One attempt to understand this finding is in terms of projection effects (different inclinations for the galaxies observed thus far). If the shells are intrinsically triaxial, their projected shape on the sky will invariably be an ellipse with an ellipticity that varies as a function of the intrinsic axial ratios, the inclination of the galaxy, and the position angle of the major axis of the hole within the plane of the galaxy. This should lead to a wide range of ellipticities. The fact that we do not see this in IC 2574 can only mean that the shells in this galaxy are intrinsically spherical.

This can be understood by considering the shapes of the rotation curves of the galaxies. IC 2574 is the only object that exhibits a rising rotation curve throughout, as is typical for dwarf galaxies. Because the rotation curve does not turn over, leading to a flat part dominated by differential rotation, there is no shear to distort and ultimately destroy any large-scale structures such as $\mathrm{H}$ i shells. For a more complete discussion on this topic, see Silich et al. (1996) and Palouš et al. (1990). Indeed, PWBR92 estimate that the amount of shear over 1 or $2 \mathrm{kpc}$ in radius, once the flat part of the rotation curve is reached in Ho II, is sufficient to stretch the holes to an axial ratio of 0.5 over a timescale of
$10^{8}$ years. IC 2574 , however, is the only object studied to date that shows solid-body rotation almost throughout the disk (MCR94), which ensures that holes stay circular until long after their creation.

There is one fly in the ointment. Why should the shells in IC 2574 be intrinsically spherical? The expansion rate perpendicular to the disk should be different from that in the disk. In the direction perpendicular to the disk, the volume density rapidly decreases, favoring faster expansion in that direction. But on the other hand, the gas that moves upward starts to feel the gravitational pull from the mass surface density below it, slowing it down. It thus seems as if nature conspires to create, at least in this particular object, holes that are near spherical in shape.

Based on the absence of elliptical holes, an interesting conclusion can be drawn concerning magnetic fields. Usually, the influence of magnetic fields is neglected in numerical studies of expanding bubbles in the ISM. However, a few analytical approaches have been put forward in recent years to investigate the effects of the evolution of shell-like structures in the presence of magnetic fields (e.g., Tomisaka 1991). The results of these investigations are that if global magnetic fields are present, they would inhibit the expansion of a supernova-driven bubble in directions perpendicular to the field lines. The expansion along the field lines would not be affected, thus leading to elliptical, elongated structures. The fact that we observe only spherical holes in IC 2574 shows that either magnetic fields play a minor role or that the magnetic fields in IC 2574 are really weak.

\section{SUMMARY}

High-resolution $\mathrm{H}$ I and $\mathrm{H} \alpha$ imaging using the VLA and the Calar Alto observatory of the dwarf galaxy IC 2574 have been presented in this paper. The main results and conclusions are:

1. IC 2574 is a gas-rich dwarf galaxy that shows a stunning amount of detail in the form of $\mathrm{H}$ I shells and holes in its ISM. These features are similar to those found in the Galaxy, the Magellanic Clouds, M31, M33, and M101 and NGC 6946, IC 10, and Ho II. From the global H I profile we derive a total $\mathrm{H}$ I mass of $9.0 \times 10^{8} M \odot$.

2. Using two independent approaches, the thickness of the H I layer of IC 2574 has been derived to be $\approx 350 \mathrm{pc}$. This implies that IC 2574, as is the case for Ho II, is a "thick" dwarf galaxy (compared with more massive spirals) which can be understood in terms of its lower gravitational potential.

3. Large-scale structures emptied of $\mathrm{H}$ I (believed to be expanding $\mathrm{H}$ I shells) leave roughly circular footprints, $\mathrm{H}$ I holes. They are prominent in the high-resolution VLA channel maps of IC 2574. In total, $48 \mathrm{H}$ I holes have been cataloged, and they are distributed across the entire galaxy. The diameters of the holes range between $100 \mathrm{pc}$ and 1200 pc. The lower limit of the sizes is due to the limit of our spatial resolution. Most of the holes are found to be expanding, with typical expansion velocities ranging between 8 and $12 \mathrm{~km} \mathrm{~s}^{-1}$. The indicative ages of the $\mathrm{H} \mathrm{I}$ holes range between 10 and $60 \times 10^{6}$ years. Typical energies required to create the observed features are of the order of 10-100 $\times 10^{50} \mathrm{ergs}$, assuming that they were created by the combined effects of stellar winds and supernova explo- 
sions. The $\mathrm{H}$ I masses that were present at the position of the holes before creation (and that might now be present on the rim of the shell) are of the order of $10-100 \times 10^{4} M_{\odot}$.

4 . In addition to the well defined holes, some large-scale $(>1000 \mathrm{pc})$, coherent features are visible in the channel maps. They might be the remainder of an older shell population.

5. The radial expansion of the holes, the indicative ages, and the energy requirements for their formation suggest that they have been created by the combined effects of stellar winds and multiple supernova explosions. Despite their large dimensions, the energy requirements are not extreme, and infall of material such as clouds is not needed to explain the observed features. However, alternative mechanisms cannot be ruled out.

6 . The shells can grow to these large dimensions because of several conditions that are fulfilled in dwarf galaxies. The volume density in the plane is low, which facilitates expansion. In the direction perpendicular to the disk, the gravitational pull is smaller than in a massive spiral. Also, because of the thick H I layer, shells are easily contained and unlikely to blow out. Lastly, solid-body rotation and a lack of spiral density waves prevent holes from being rapidly destroyed.

7. A comparison with other galaxies studied in similar detail so far (M31, M33, and Ho II) shows that the size distribution of $\mathrm{H}$ I holes found in a galaxy is related to its Hubble type in the following way. The size of the largest $\mathrm{H} \mathrm{I}$ shells is inversely proportional to the global gravitational potential (and hence mass surface density). The shells in dwarf galaxies show lower expansion velocities, most likely because, in the absence of shear (differential rotation), the shells are preserved much longer. The energies needed to create these structures, however, are found to be roughly the same for all types of galaxies. If we follow the conventional view that star forming regions are responsible for the structures found in $\mathrm{H} \mathrm{I}$, the energy output of a typical starforming region does not seem to be related to the host galaxy.

8. The fact that virtually all holes in IC 2574 are circular in shape is a major difference with the other galaxies studied in comparable detail and can be explained, again, by the absence of shear and spiral density waves in IC 2574. This finding also indicates that magnetic fields play only a minor role in shaping expanding holes or that the magnetic fields in IC 2574 are weak.

9. Current star formation, as traced by $\mathrm{H} \alpha$ emission, is predominantly found along the rims of the larger $\mathrm{H}$ i holes, indicating propagating star formation. A detailed compari- son of the $\mathrm{H} \alpha$ and the $\mathrm{H}$ I properties shows that, on a global scale, star formation is only present if the $\mathrm{H}$ I surface density reaches values larger then $10^{21} \mathrm{~cm}^{-2}$ (on a linear scale of $\approx 95 \mathrm{pc}$ ).

In summary, our results on IC 2574 confirm the findings by PWBR92 and show that Ho II is not a special case. Our analysis, and the comparison with other galaxies studied in similar detail suggest that there is a trend of hole characteristics with Hubble type (or mass). If the conclusion that the energy output of a SF region is independent of the host galaxy holds, then it follows that there is a lower mass limit for the formation of dwarf galaxies. In other words, if the mass of a galaxy falls below a certain threshold, the first burst of star formation will tear the whole galaxy apart (see also, e.g., Puche \& Westpfahl 1994). Mac Low \& Ferrara (1999) predict that this lower mass limit will be near $10^{6}$ $M_{\odot}$, which is well below the mass of the objects dealt with in this paper.

The authors are indebted to Mordecai-Marc Mac Low for providing them with a model data cube of a turbulent ISM in advance of publication and to Johan van Horebeek for valuable advice regarding some of the statistical techniques employed. F. W. acknowledges the Deutsche Forschungsgemeinschaft for the award of a fellowship associated with the Graduiertenkolleg on The Magellanic Clouds and other Dwarf Galaxies and would like to thank Uli Klein for continuous support and Neb Duric for fruitful discussions. F. W. appreciates the help of the staff of the Array Operation Center of the NRAO during his stay in Socorro, New Mexico and also wants to thank the staff of the Calar Alto observatory in Spain for excellent working conditions. F. W. is grateful to Jan Palouš for illuminating discussions during his stays at the Academy of Science in Prague regarding the numerical simulations of holes and shells. E. B. acknowledges a grant awarded by CONACyT (grant number 0460P-E) and is grateful for the support by the Graduiertenkolleg, without which this collaborative effort would not have been possible. Last but not least, we would like to thank Klaas de Boer for carefully reading the manuscript and an anonymous referee for useful suggestions. This research has made use of the NASA/IPAC Extragalactic Database (NED), which is operated by the Jet Propulsion Laboratory, Caltech, under contract with the National Aeronautics and Space Administration (NASA), NASA's Astrophysical Data System Abstract Service (ADS), and NASA's SkyView.

\section{REFERENCES}

Arsenault, R., \& Roy, J. R. 1986, AJ, 92, 567

Baars, J. W. M., Genzel, R., Pauliny-Toth, I. I. K., \& Witzel, A. 1977, A\&A, 61,99

Bottema, R., Shostak, G. S., \& van der Kruit, P. C. 1986, A\&A, 167, 34

Briggs, D. 1995, Ph.D. thesis, New Mexico Inst. Mining and Technol.

Brinks, E. 1981, A\&A, 95, L1

Brinks, E., \& Bajaja, E. 1986, A\&A, 169, 14

Chevalier, R. A. 1974, ApJ, 188, 501

Chyzy, K. T., Kohle, S., Beck, R., Klein, U., \& Urbanik, M. 1998, in Proc. Bonn/Bochum Graduiertenkolleg Workshop, The Magellanic Clouds and Other Dwarf Galaxies, ed. T. Richtler \& J. M. Braun (Bonn: Shaker), 237

Clark, B. G. 1980, A\&A, 89, 377

Cox, D. P., \& Smith, B. W. 1974, ApJ, 189, L105

Deeg, H.-J., Brinks, E., Duric, N., Klein, U., \& Skillman, E. 1993, ApJ, 410, 626

Deul, E. R., \& den Hartog, R. H. 1990, A\&A, 229, 362 de Vaucouleurs, G., de Vaucouleurs, A., Corwin, H. G., Buta, R. J., Paturel, G., \& Fouqué, P. 1991, Third Reference Catalogue of Bright Galaxies (New York: Springer) (RC3)

Devereux, N. A., Duric, N., \& Scowen, P. A. 1997, AJ, 113, 236

Dressel, L. L., \& Condon, J. J. 1976, ApJS, 31, 187

Drissen, L., Roy, J.-R., \& Moffat, A. F. J. 1993, AJ, 106, 1460

Efremov, Y. N., \& Elmegreen, B. G. 1998, MNRAS, 299, 643

Efremov, Y. N., Elmegreen, B. G., \& Hodge, P. W. 1998, ApJ, 501, L163

Elmegreen, B. G. 1994, ApJ, 427, 384

Field, G. B., Goldsmith, D. W., \& Habing, H. J. 1969, ApJ, 155, L149

Gerritsen, J. P. E., \& Icke, V. 1997, A\&A, 325, 972

Heiles, C. 1979, ApJ, 229, 533

.1984, ApJS, 55, 585 1990, ApJ, 354, 483

Högbom, J. A. 1974, A\&AS, 15, 417

Huchtmeier, W. K., \& Richter, O. G. 1988, A\&A, 203, 237

Hunter, D. A., Elmegreen, B. G., \& Baker, A. L. 1998, ApJ, 493, 595 
Jörsäter, S., \& van Moorsel, G. A. 1995, AJ, 110, 2037

Kamphuis, J. J. 1993, Ph.D. thesis, Univ. Groningen

Kellmann, S. A. 1972, ApJ, 175, 353

Kennicutt, R. C. 1988, ApJ, 334, 144 1989, ApJ, 344, 685

Kim, S., Staveley-Smith, L., Dopita, M. A., Freeman, K. C., Sault, R. J., Kesteven, M. J., \& McConnell, D. 1998, ApJ, 503, 674

Loeb, A., \& Perna, R. 1998, ApJ, 503, L35

Mac Low, M.-M., \& Ferrara, A. 1999, ApJ, 513, 142

Mac Low, M.-M., Klessen, R. S., Burkert, A., \& Smith, M. D. 1998, Phys. Rev. Lett., 80, 2754

Martimbeau, N., Carignan, C., \& Roy, J.-R. 1994, AJ, 107, 543 (MCR94)

McCray, R., \& Snow, T. P. Jr. 1979, ARA\&A, 17, 213

McKee, C. F. \& Ostriker, J. P. 1977, ApJ, 218, 148

Miller, B. W., \& Hodge, P. 1994, ApJ, 427, 656 (MH94) 1996, ApJ, 458, 467

Oey, M. S., \& Clarke, C. J. 1997, MNRAS, 289, 570

Palouš, J., Franco, J., \& Tenorio-Tagle, G. 1990, A\&A, 227, 175

Palouš, J., Tenorio-Tagle, G., \& Franco, J. 1994, MNRAS, 270, 75

Peimbert, M., Rayo, J. F., \& Torres-Peimbert, S. 1975, Rev. Mexicana Astron. Astrofis., 1, 289

Puche, D., \& Westpfahl, D. 1994, in ESO/OHP Conf. and Workshop Proc. 49, Dwarf Galaxies, ed. G. Meylan \& P. Prugniel (Garching: ESO), 273

Puche, D., Westpfahl, D., Brinks, E., \& Roy, J.-R. 1992, AJ, 103, 1841 (PWBR92)

Radice, L. A., Salzer, J. J., \& Westpfahl, D. J. 1995, AAS, 186, 4908

Rhode, K. L., Salzer, J. J., \& Westpfahl, D. J. 1999, AJ, 118, in print

Rohlfs, K., \& Wilson, T. L. 1996, Tools of Radio Astronomy (New York: Springer)
Rots, A. H. 1980, A\&AS, 41, 189

Sage, L. J., Salzer, J. J., Loose, H.-H., \& Henkel, C. 1992, A\&A, 265, 19

Seielstad, G. A., \& Wright, M. C. H. 1973, ApJ, 184, 343

Silich, S. A., Franco, J., Palouš, J., \& Tenorio-Tagle, G. 1996, ApJ, 468, 722

Skillman, E. D. 1987, in NASA Conf. Pub. 2466, Star Formation in Galaxies, ed. C. J. Lonsdale Persson (Washington: NASA), 263

Staveley-Smith, L., Davies, R. D., \& Kinman, T. D. 1992, MNRAS, 258, 334

Staveley-Smith, L., Sault, R. J., Hatzidimitrou, D., Kesteven, M. J., \& McConnell, D. 1997, MNRAS, 289, 225

Stewart, S. G., et al. 1999, ApJ, submitted

Tenorio-Tagle, G., \& Bodenheimer, P. 1988, ARA\&A, 26, 145

Tenorio-Tagle, G., Franco, J., Bodenheimer, P., \& Rózyczka, M. 1987, A\&A, 179, 219

Tikhonov, N. A., Karachentsev, I. D., Bilkina, B. I., \& Georgiev, T. B. 1991, A\&AS, 89, 1

Tomisaka, K. 1991, in IAU Symp. 144, The Interstellar Disk-Halo Connection in Galaxies, ed. H. Bloemen (Dordrecht: Kluwer), 407

Tomita, A., Ohta, K., Nakanishi, K., Takeuchi, T. T., \& Saito, M. 1998, AJ, 116,131

Toomre, A. 1964, ApJ, 139, 1217

van der Hulst, J. M. 1996, in ASP Conf. Ser. 106, The Minnesota Lectures on Extragalactic Neutral Hydrogen, ed. E. D. Skillman (San Francisco: ASP), 47

van der Hulst, J. M., \& Sancisi, R. 1988, AJ, 95, 1354

van der Kruit, P. C. 1981, A\&A, 99, 298

Walter, F., Brinks, E., Duric, N., Kerp, J., \& Klein, U. 1998, ApJ, 502, L143

Walter, F., Brinks, E., Duric, N., \& Klein, U. 1997, AJ, 113, 2031

Wilcots, E. M., \& Miller, B. W. 1998, AJ, 116, 2363 